\title{
Determination of the Higgs CP-mixing angle in the tau decay channels at the LHC including the Drell-Yan background
}

\author{
Stefan Berge $^{\mathrm{a}}$, Werner Bernreuther ${ }^{\mathrm{b}}$, Sebastian Kirchner ${ }^{\mathrm{c}}$ \\ Institut für Theoretische Physik, RWTH Aachen University, 52056 Aachen, Germany
}

Received: 8 August 2014 / Accepted: 31 October 2014 / Published online: 26 November 2014

(c) The Author(s) 2014. This article is published with open access at Springerlink.com

\begin{abstract}
We investigate how precisely the CP nature of the $125 \mathrm{GeV}$ Higgs-boson resonance $h$ can be unraveled at the LHC in its decays to $\tau$ pairs, $h \rightarrow \tau^{-} \tau^{+}$. We use a method which allows one to determine the scalar-pseudoscalar Higgs mixing angle $\phi_{\tau}$ in this decay mode. This mixing angle can be extracted from the distribution of a signed angle, denoted by $\varphi_{\mathrm{CP}}^{*}$, which we analyze for the major charged-prong $\tau$ decays. For definiteness, we consider Higgs-boson production by gluon fusion at NLO QCD. We take into account also the irreducible background from Drell-Yan production, $Z^{*} / \gamma^{*} \rightarrow \tau \tau$, at NLO QCD. We compute, for the signal and background reactions, angular and energy correlations of the charged prongs and analyze which type of cuts suppress the Drell-Yan background. An important feature of this background is that its contribution to the distribution of our observable $\varphi_{\mathrm{CP}}^{*}$ is a flat line, also at NLO QCD. By separating the Drell-Yan $\tau$ events into two different sets, two different non-trivial $\varphi_{\mathrm{CP}}^{*}$ distributions are obtained. Based on this observation we propose to use these sets for calibration purposes. By Monte Carlo simulation we study also the effect of measurement uncertainties on this distribution. We estimate that the Higgs mixing angle $\phi_{\tau}$ can be determined with our method to a precision of $\Delta \phi_{\tau} \simeq 14^{\circ}\left(5^{\circ}\right)$ at the highluminosity LHC (14 TeV) with an integrated luminosity of $500 \mathrm{fb}^{-1}\left(3 \mathrm{ab}^{-1}\right)$.
\end{abstract}

\section{Introduction}

The Large Hadron Collider (LHC) has had its first major triumph with the discovery of a new, electrically neutral boson $h$ with mass $m_{h} \simeq 125 \mathrm{GeV}$ by the ATLAS and CMS experiments $[1,2]$. According to present experimental knowledge

\footnotetext{
a e-mail: berge@physik.rwth-aachen.de

be-mail: breuther@physik.rwth-aachen.de

c e-mail: kirchner@physik.rwth-aachen.de
}

on the spin and parity of $h[3,4]$ and its couplings to gauge bosons and quarks and leptons [3-11], the properties of this boson agree with those of the Higgs boson predicted by the Standard Model (SM) of particle physics. In particular, the decay of $h$ to a pair of $\tau$ leptons was recently established $[8,10]$.

Nevertheless, much more experimental analysis is required for completely unraveling the properties of this resonance. In particular, although the LHC data strongly prefer that $h$ is a $J^{P}=0^{+}$state, it is not yet excluded with high probability that $h$ has a pseudoscalar component. How the spin and $C P$ quantum number of a neutral Higgs-like boson can be pinned down at a hadron collider or at a future (linear) $e^{+} e^{-}$collider has been investigated in numerous papers, including [12-75].

In this paper we elaborate on a method for determining the $C P$ nature of a Higgs-like resonance at the LHC in its decays to $\tau$ leptons which has been developed in a series of papers $[48,51,56,67]$ both for Higgs production at the LHC and in $e^{+} e^{-}$collisions. Our approach is based on the distribution of a signed angle $\varphi_{\mathrm{CP}}^{*}$ between the decay planes of the charged-prong decays $\tau^{-} \rightarrow a^{-}$and $\tau^{+} \rightarrow a^{\prime+}$ in the $a^{-} a^{\prime+}$ zero-momentum frame. We apply this method to the $125 \mathrm{GeV}$ resonance $h$. We assume that $h$ is a mixture of a CP-even and CP-odd state with the CP-odd admixture being smaller than the CP-even one. This assumption is in accord with the analysis of present data, cf. for instance [7680]. We investigate also the contribution of the irreducible background $Z^{*} / \gamma^{*} \rightarrow \tau \tau$ to the $\varphi_{\mathrm{CP}}^{*}$ distribution. While the signal contribution to this distribution shows a characteristic dependence on $\cos \left(\varphi_{\mathrm{CP}}^{*}-2 \phi_{\tau}\right)$, where the angle $\phi_{\tau}$ describes the mixing of the scalar and pseudoscalar Higgs component which couple to $\tau$ leptons (see Sect. 2), we find that the background contribution is flat. We point out how the background events $Z^{*} / \gamma^{*} \rightarrow \tau \tau$, which are numerous at the LHC, can be used by experiments for calibrating the measurements of the distribution of the angle $\varphi_{\mathrm{CP}}^{*}$. Moreover, we consider the two- 
dimensional helicity-angle and energy distributions of the charged prongs from $\tau^{\mp}$ decay, which differ for $h \rightarrow \tau \tau$ and $Z^{*} / \gamma^{*} \rightarrow \tau \tau$ because of the different spins of the bosons, and analyze whether appropriate cuts can enhance the signalto-background ratio. Furthermore, we estimate the precision with which the scalar-pseudoscalar mixing angle $\phi_{\tau}$ might be measured at the LHC (14 TeV).

The paper is organized as follows. In the next section we briefly describe the $\tau$-decay modes and decay density matrices which are used in this analysis. In Sects. 3 and 4 we exhibit the helicity-angle distributions of the charged prongs from $h \rightarrow \tau \tau$ decay at the LHC. We recall the definition of the angle $\varphi_{\mathrm{CP}}^{*}$ and its distribution in $h \rightarrow \tau \tau$ with subsequent decays to charged prongs, which allows one to determine the CP-mixing angle $\phi_{\tau}$. In Sect. 5 we analyze the helicity and azimuthal angle distributions of the charged prongs for Drell-Yan production of $\tau^{-} \tau^{+}$at the LHC. In particular, we elaborate on the distribution of the angle $\varphi_{\mathrm{CP}}^{*}$ in $Z^{*} / \gamma^{*} \rightarrow \tau \tau \rightarrow \pi^{-} \pi^{+}$. The discussion in this section is based on the leading-order distributions. We have computed these distributions also at next-to-leading order (NLO) QCD. The results are given in Sect. 6 and are compared with the corresponding distributions for inclusive Higgs production at the LHC, which we computed at NLO QCD by including differential Higgs-boson distributions obtained with the computer code MCFM [81,82] into our Monte Carlo program. We analyze how appropriate cuts on the polar angle distributions of the charged prongs, respectively, associated cuts on their energies can reduce the irreducible background. Moreover, we study the impact of measurement uncertainties on these distributions by Monte Carlo simulation. Finally we estimate the precision with which the scalar-pseudoscalar mixing angle $\phi_{\tau}$ might be measured at the LHC (14 TeV). We summarize in Sect. 7.

\section{Higgs-boson production and decay to $\tau \tau$}

Our method to determine the $\mathrm{CP}$ nature of the $125 \mathrm{GeV}$ resonance $h$ in its $\tau \tau$ decays, which will be described in Sect. 4, can be applied to any $h$ production mode, but for definiteness we consider $h$ production at the LHC by gluon gluon fusion,

$p p \rightarrow h+X$

We consider the decay mode $h \rightarrow \tau^{-} \tau^{+}$with subsequent decays

$h \rightarrow \tau^{-} \tau^{+} \rightarrow a^{-} a^{\prime+}+X$,

where $a^{ \pm}, a^{ \pm} \in\left\{e^{ \pm}, \mu^{ \pm}, \pi^{ \pm}, a_{1}^{L, T, \pm}\right\}$ and $X$ denotes neutrinos and $\pi^{0}$. We take into account the main 1- and 3charged-prong $\tau$ decay modes:

$\tau \rightarrow l+v_{l}+v_{\tau}$

$$
\begin{aligned}
& \tau \rightarrow \pi+v_{\tau}, \\
& \tau \rightarrow \rho+v_{\tau} \rightarrow \pi+\pi^{0}+v_{\tau}, \\
& \tau \rightarrow a_{1}+v_{\tau} \rightarrow \pi+2 \pi^{0}+v_{\tau}, \\
& \tau \rightarrow a_{1}^{L, T}+v_{\tau} \rightarrow 2 \pi^{ \pm}+\pi^{\mp}+v_{\tau} .
\end{aligned}
$$

We call the decay mode (7) also '1-prong', because the 4momentum of $a_{1}^{ \pm}$can be obtained from the measured 4momenta of the 3 charged pions. The longitudinal $(L)$ and transverse $(T)$ helicity states of the $a_{1}$ resonance can be separated by using known kinematic distributions [83-86].

The dynamics of the above $\tau$ decays is, to the precision relevant for our purposes, known Standard Model physics. The interaction of a Higgs boson $h$ of arbitrary $C P$ nature to $\tau$ leptons is described by the Yukawa Lagrangian

$\mathscr{L}_{Y}=-\left(\sqrt{2} G_{F}\right)^{1 / 2} m_{\tau}\left(a_{\tau} \bar{\tau} \tau+b_{\tau} \bar{\tau} i \gamma_{5} \tau\right) h$,

where $G_{F}$ denotes the Fermi constant and $a_{\tau}, b_{\tau}$ are the reduced dimensionless $\tau$ Yukawa coupling constants. Instead of (8), we use in the following the equivalent parameterization,

$\mathscr{L}_{Y}=-g_{\tau}\left(\cos \phi_{\tau} \bar{\tau} \tau+\sin \phi_{\tau} \bar{\tau} i \gamma_{5} \tau\right) h$,

where $g_{\tau}$ is the effective strength of the $\tau$-Yukawa interaction and $\phi_{\tau}$ describes the degree of mixing of the scalar and pseudoscalar Higgs component which couple to $\tau$ leptons.

$g_{\tau}=\left(\sqrt{2} G_{F}\right)^{1 / 2} m_{\tau} \sqrt{a_{\tau}^{2}+b_{\tau}^{2}}, \quad \tan \phi_{\tau}=\frac{b_{\tau}}{a_{\tau}}$.

As to the mixing angle $\phi_{\tau}$, we remark the following. It is in general not universal, but specific to the $\tau$-Yukawa interaction. The reduced Yukawa couplings $a_{f}, b_{f}$ to quarks and leptons $f$ are model-dependent. As an example one may consider type-II two-Higgs doublet extensions of the Standard Model, where the SU(2) Higgs doublet $\Phi_{2}$ is coupled to the right-chiral $u$-type quarks and the other doublet $\Phi_{1}$ is coupled to right-chiral $d$-type quarks and charged leptons. Referring to the model described for instance in [87] one obtains in this case that $a_{f}$ and likewise $b_{f}$ are identical for $d$-type quarks and charged leptons, while they differ in general for $u$-type quarks. Defining $\tan \phi_{t}=b_{t} / a_{t}$, where $a_{t}, b_{t}$ are the reduced Yukawa couplings of the top quark, one gets $\tan \phi_{\tau}=$ $\tan \alpha \tan \beta \tan \phi_{t}$, where $\tan \beta=v_{2} / v_{1}$ is the ratio of the vacuum expectation values of the Higgs doublets $\Phi_{2}$ and $\Phi_{1}$. For notational simplicity we call $\phi_{\tau}$ the Higgs mixing angle.

For the SM Higgs boson, which is CP-even, one has $g_{\tau}=\left(\sqrt{2} G_{F}\right)^{1 / 2} m_{\tau}$ and $\phi_{\tau}=0$. The ATLAS and CMS results on the $125 \mathrm{GeV}$ resonance $h$ exclude that it is a pure pseudoscalar. In the following we investigate how precisely a possible pseudoscalar component of $h$, i.e. $\sin \phi_{\tau} \neq 0$, can be determined in its $\tau$ decays at the LHC by means of the observables defined below.

The observables that we use $[14,28,48,51,56,67]$ are based on $\tau$-spin correlations. The charged prongs, i.e., the 
charged lepton $l=e, \mu$ in (3), the charged pion in (4)-(6), and the $a_{1}^{L, T}$ serve as $\tau$-spin analyzers. The normalized distributions of polarized $\tau$ decays to a $\pi^{\mp}$, a charged lepton $l=e, \mu$, a charged $\rho$ or $a_{1}$, and to a charged pion via $\rho$ and $a_{1}$ decay are, in the $\tau$ rest frame, of the form

$$
\begin{aligned}
& \Gamma_{a}{ }^{-1} \mathrm{~d} \Gamma\left(\tau^{\mp}\left(\hat{\mathbf{s}}^{\mp}\right) \rightarrow a^{\mp}\left(q^{\mp}\right)+X\right) \\
& \quad=n\left(E_{\mp}\right)\left[1 \pm b\left(E_{\mp}\right) \hat{\mathbf{s}}^{\mp} \cdot \hat{\mathbf{q}}^{\mp}\right] d E_{\mp} \frac{d \Omega_{\mp}}{4 \pi} .
\end{aligned}
$$

Here the $\hat{\mathbf{s}}^{\mp}$ denote the normalized spin vectors of the $\tau^{\mp}$ and $E \mp$ and $\hat{\mathbf{q}}^{\mp}$ are the energies and directions of flight of $a^{\mp}=$ $l^{\mp}, \pi^{\mp}$ in the respective $\tau$ rest frames. The spectral functions $n$ and $b$ are given in [56]. The function $b\left(E_{\mp}\right)$ encodes the $\tau$ spin analyzing power of the particle $a^{\mp}$. The $\tau$-spin analyzing power is maximal for the direct decays to pions, $\tau^{\mp} \rightarrow \pi^{\mp}$, and for $\tau^{\mp} \rightarrow a_{1}^{L, T, \mp}$. (We recall that the $\tau$-spin analyzing power of $a_{1}^{L-}$ and $a_{1}^{T-}$ is +1 and -1 , respectively.) For the other decays, the $\tau$-spin analyzing power of $l^{\mp}$ and $\pi^{\mp}$ depends on the energy of these particles. It can be optimized by judiciously chosen energy cuts.

\section{Distributions for Higgs production and decay to $\tau \tau$ at the LHC}

The hadronic differential cross section $d \sigma$ for Higgs production at the LHC is given as a convolution of parton distribution functions and the partonic differential cross section $d \hat{\sigma}_{i j}$ for the production of $h$ by partons $i$ and $j$ and subsequent $h$ decay. For the decays (2) of $h$ to $\tau$ leptons $d \hat{\sigma}_{i j}$ factorizes into a product of the squared $h$ production and decay matrix elements, as long as one neglects higher-order electroweak corrections that connect the production and $\tau$-decay stage of $h$. The $125 \mathrm{GeV}$ resonance $h$ is narrow, $\Gamma_{h}<4.2 \Gamma_{h}^{S M}$ at $95 \% \mathrm{CL}$ [88] where $\Gamma_{h}^{S M}=4.29 \mathrm{MeV}$ (see, for instance [89]). Therefore we can use the narrow width approximation for $h$. In the following we are interested in the angular correlations of the charged prongs $a^{+}$and $a^{-}$in the decays (2). The characteristic features of these correlations depend only on the $C P$ nature of $h$, but not on the details of its production. Therefore we exhibit these correlations for the case of inclusive Higgs production $i j \rightarrow h+X \rightarrow \tau^{-} \tau^{+}+X \rightarrow a^{-} a^{\prime+}+X$ (which is dominated by gluon fusion). The structure of these correlations applies also to other processes, for instance $h+$ jet production or $h$ production by vector boson fusion.

We choose a right-handed coordinate frame where the $\tau^{-}$ direction of flight $\hat{\mathbf{k}}$ in the $\tau^{-} \tau^{+}$zero-momentum frame $(\tau \tau$ ZMF) defines the $z$ axis. The $\tau^{ \pm}$rest frames are connected with the $\tau \tau$ ZMF by rotation-free Lorentz boosts. In Eq. (13), $\theta_{\mp}=L\left(\hat{\mathbf{k}}, \hat{\mathbf{q}}_{\mp}\right)$ are the polar angles of $a^{-}$and $a^{\prime+}$, where $\hat{\mathbf{q}}_{\mp}$ are the directions of flight of $a^{-}$and $a^{\prime+}$ in the $\tau^{\mp}$ rest frame, respectively, and $\varphi=\phi_{-}-\phi_{+}, \quad 0 \leq \varphi \leq 2 \pi$,

is the difference of their azimuthal angles. With (9) and (11) we obtain for the differential partonic cross section at leading order

$$
\begin{aligned}
d \hat{\sigma}_{i j}= & \frac{g_{\tau}^{2} m_{h}}{128 \pi^{3} s \Gamma_{h}} \bar{\sum}|M(i j \rightarrow h)|^{2} \mathrm{Br}_{\tau^{-} \rightarrow a^{-}} \mathrm{Br}_{\tau^{+} \rightarrow a^{\prime+}} \\
& \times d \Omega_{\tau} d E_{-} d \Omega_{-} d E_{+} d \Omega_{+} n\left(E_{+}\right) n\left(E_{-}\right) \\
& \times\left[1+b\left(E_{+}\right) b\left(E_{-}\right)\left(\cos \theta_{+} \cos \theta_{-}\right.\right. \\
& \left.\left.-\sin \theta_{+} \sin \theta_{-} \cos \left(\varphi-2 \phi_{\tau}\right)\right)\right] .
\end{aligned}
$$

Here, $\sqrt{s}$ is the partonic center-of-mass energy, $m_{h}$ is the mass of $h$, and $\phi_{\tau}$ is the Higgs mixing angle defined in (9). Moreover, we have put in (13) the $\tau$ velocity $\beta_{\tau}$ in the $h$ rest-frame equal to one.

The angular correlations in (13) reflect the $\tau$ spin correlations induced in the decay of $h$. Integrating Eq. (13) with respect to $d \Omega_{\tau} d \cos \theta_{-} d \cos \theta_{+}$, the differential partonic cross section takes the form

$$
\begin{aligned}
d \hat{\sigma}_{i j}= & \frac{g_{\tau}^{2} m_{h}}{8 \pi^{2} s \Gamma_{h}} \bar{\sum}|M(i j \rightarrow h)|^{2} \mathrm{Br}_{\tau^{-} \rightarrow a^{-}} \mathrm{Br}_{\tau^{+} \rightarrow a^{\prime+}} \\
& \times d \varphi d E_{-} d E_{+} n\left(E_{+}\right) n\left(E_{-}\right) \\
& \times\left[1-b\left(E_{+}\right) b\left(E_{-}\right) \frac{\pi^{2}}{16} \cos \left(\varphi-2 \phi_{\tau}\right)\right] .
\end{aligned}
$$

Also this distribution encodes the CP nature of $h$. It was shown in [51,67] that the difference $\varphi$ of the azimuthal angles, which is equal to the angle between the signed normal vectors of the $\tau^{-} \rightarrow a^{-}$and $\tau \rightarrow a^{\prime+}$ decay planes, can actually be measured in the zero-momentum frame of the charged prongs $a^{-}$and $a^{\prime+}$. This has the big advantage that the $\tau^{\mp}$ momenta need not be reconstructed in experiments.

\section{Observables}

Our method to determine the $\mathrm{CP}$ nature of a spin-zero resonance $h$ in its decays (2) has been described in detail in $[51,56,67]$. We recall here its salient features. It requires the measurement of the 4-momenta of the charged prongs $a^{-}$, $a^{\prime+}$ and their impact parameter vectors $\mathbf{n}_{\mp}$ in the laboratory frame. The corresponding unit vectors are denoted by $\hat{\mathbf{n}}_{\mp}$. The 4-vectors $n_{\mp}^{\mu}=\left(0, \hat{\mathbf{n}}_{\mp}\right)$ are boosted into the $a^{-} a^{\prime+} \mathrm{ZMF}$ and the spatial parts of the resulting 4-vectors $n_{\mp}^{* \mu}$ are decomposed into their normalized components $\hat{n}_{\|}^{* \mp}$ and $\hat{n}_{\perp}^{* \mp}$ which are parallel and perpendicular to the respective 3-momentum of $a^{-}$and $a^{\prime+}$. With the 'unsigned' normal vectors $\hat{n}_{\|}^{* \mp}$ one determines the 'unsigned' angle $\varphi^{*}$ between the $\tau^{-} \rightarrow a^{-}$ and $\tau \rightarrow a^{\prime+}$ decay planes in the $a^{-} a^{\prime+}$ ZMF:

$\varphi^{*}=\arccos \left(\hat{\mathbf{n}}_{\perp}^{*+} \cdot \hat{\mathbf{n}}_{\perp}^{*-}\right), \quad 0 \leq \varphi^{*} \leq \pi$. 


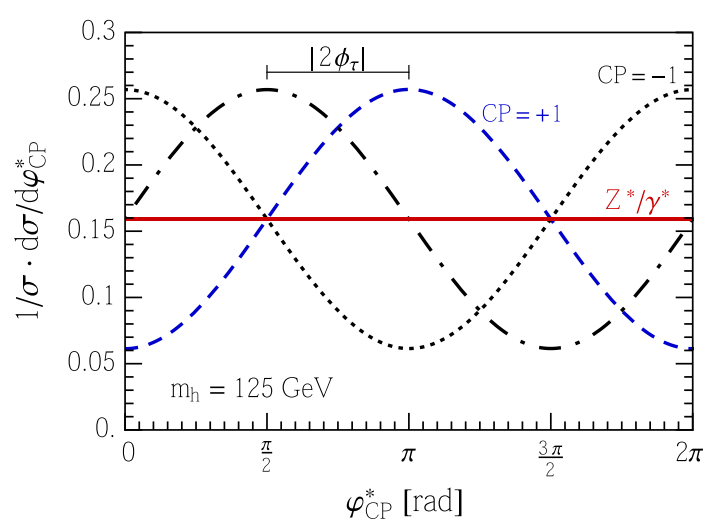

Fig. 1 Normalized $\varphi_{\mathrm{CP}}^{*}$ distribution for the case where both $\tau^{-}$and $\tau^{+}$decay to $\pi \nu$. The blue dashed line is the distribution for a $C P$-even Higgs boson $\left(\phi_{\tau}=0\right)$ and the black long-dash dotted line corresponds to a $C P$ mixture with $\phi_{\tau}=-\frac{\pi}{4}$. In addition, the distribution for a $C P$ odd Higgs boson $\left(\phi_{\tau}= \pm \pi / 2\right.$, black dotted line $)$ is also shown. The solid red line is the distribution due to the $Z^{*} / \gamma^{*} \rightarrow \tau^{+} \tau^{-}$background, cf. Sect. 5

The simultaneous measurement of (15) and of the $C P$-odd and $T$-odd triple correlation

$\mathscr{O}_{\mathrm{CP}}^{*}=\hat{\mathbf{p}}_{-}^{*} \cdot\left(\hat{\mathbf{n}}_{\perp}^{*+} \times \hat{\mathbf{n}}_{\perp}^{*-}\right)$,

where $\hat{\mathbf{p}}_{-}^{*}$ is the normalized $a^{\prime-}$ momentum in the $a^{-} a^{\prime+}$ $\mathrm{ZMF}$, allows one to determine a 'signed' angle (in the range 0 to $2 \pi$ ) between the $\tau^{-} \rightarrow a^{-}$and $\tau \rightarrow a^{\prime+}$ decay planes in the $a^{-} a^{\prime+} \mathrm{ZMF}$, which is denoted by $\varphi_{\mathrm{CP}}^{*}$, by the following prescription:

$\varphi_{\mathrm{CP}}^{*}=\left\{\begin{array}{lll}\varphi^{*} & \text { if } \quad \mathscr{O}_{\mathrm{CP}}^{*} \geq 0, \\ 2 \pi-\varphi^{*} & \text { if } \quad \mathscr{O}_{\mathrm{CP}}^{*}<0 .\end{array}\right.$

The distribution of (17) is given by (14) with $\varphi \rightarrow \varphi_{\mathrm{CP}}^{*}$. In terms of this angle, the triple correlation (16) is given by $\sin \varphi_{\mathrm{CP}}^{*}$.

The distribution of (17) allows for an unambiguous determination of the CP nature of $h$, that is, of the Higgs mixing angle $\phi_{\tau}$. For illustration, the distribution of $\varphi_{\mathrm{CP}}^{*}$ is shown in Fig. 1 for the decay mode $\tau^{-} \tau^{+} \rightarrow \pi^{+} \pi^{-}+2 v$ for a $\mathrm{CP}$-even and CP-odd Higgs boson and a $\mathrm{CP}$ mixture.

By fitting the function $f=u \cos \left(\varphi_{\mathrm{CP}}^{*}-2 \phi_{\tau}\right)+\mathrm{v}$ to the measured differential distributions of $\varphi_{\mathrm{CP}}^{*}$ for the respective final states $a a^{\prime}$, one can determine the mixing angle $\phi_{\tau}$. The function $f$ is subject to the constraint $\int_{0}^{2 \pi} d \varphi_{\mathrm{CP}}^{*} f=2 \pi \mathrm{v}=$ $\sigma_{a a^{\prime}}$, where $\sigma_{a a^{\prime}}$ is the $h$-production cross section including the respective decay branching fractions. For a certain final state $a a^{\prime}$ the estimate of the statistical uncertainty of $\varphi_{\mathrm{CP}}^{*}$ depends on the values of the associated parameters $u$ and $\mathrm{v}$. Because $u$ and $\mathrm{v}$ describe the unnormalized distributions, it is more convenient to compare the different channels with a normalized quantity. The following asymmetry turns out to suit this purpose [67]:

$$
\begin{aligned}
A^{a a^{\prime}}= & \frac{1}{\sigma_{a a^{\prime}}} \int_{0}^{2 \pi} d \varphi_{\mathrm{CP}}^{*}\left\{d \sigma_{a a^{\prime}}\left(u \cos \left(\varphi_{\mathrm{CP}}^{*}-2 \phi_{\tau}\right)>0\right)\right. \\
& \left.-d \sigma_{a a^{\prime}}\left(u \cos \left(\varphi_{\mathrm{CP}}^{*}-2 \phi_{\tau}\right)<0\right)\right\} \\
= & \frac{-4 u}{2 \pi \mathrm{v}}
\end{aligned}
$$

This asymmetry can also be computed from (13), (14). In the absence of cuts, one obtains

$$
A^{a a^{\prime}}=\frac{\pi}{8} \frac{\int d E_{a^{\prime}+} d E_{a^{-}} n\left(E_{a^{+}}\right) n\left(E_{a^{-}}\right) b\left(E_{a^{+}}\right) b\left(E_{a^{-}}\right)}{\int d E_{a^{\prime}} d E_{a^{-}} n\left(E_{a^{\prime}}\right) n\left(E_{a^{-}}\right)} .
$$

Equations (18), (19) show that the values of $A^{a a^{\prime}}$ are independent of the mixing angle $\phi_{\tau}$ but do depend on the product of the $\tau$-spin analyzing powers of $a$ and $a^{\prime}$. The larger $A^{a a^{\prime}}$ the smaller the statistical error $\Delta \phi_{\tau}$ in this decay channel, for a given number of events. The $\tau$-spin analyzing power, and thus $A^{a a^{\prime}}$, is maximal for the direct decays $\tau^{\mp} \rightarrow \pi^{\mp}$ and for $\tau^{\mp} \rightarrow a_{1}^{L, T \mp}$. The $\tau$-spin analyzing power of the charged lepton in $\tau^{\mp} \rightarrow l^{\mp}$ and of the charged pion from $\tau^{\mp} \rightarrow \rho^{\mp}$ and $\tau^{\mp} \rightarrow a_{1}^{\mp}$ can be enhanced by applying an appropriate cut on the energy of the lepton and the pion, respectively $[56,67]$.

The background from Drell-Yan processes to Higgs production at the LHC affects the respective distribution $d \sigma / d \varphi_{\mathrm{CP}}^{*}$. As will be shown below, this background contribution to the $\varphi_{\mathrm{CP}}^{*}$ distribution is flat for all charged prongs $a, a^{\prime}$ if integrated over the full phase space of the final states from the $\tau^{\mp}$ decays. Of course, this contribution decreases the height of the normalized distribution and thus the magnitude of the asymmetry (18). In the next sections we investigate which cuts may be used to significantly suppress this background.

\section{Drell-Yan production of $\tau^{-} \tau^{+}$}

Background reactions to the $h \rightarrow \tau^{-} \tau^{+}$signal include production of QCD multijets, $t \bar{t}$, single top, $W+$ jets, $Z^{*} / \gamma^{*}+$ jets, $W W, W Z$, and $Z Z$. The Drell-Yan process $Z^{*} / \gamma^{*} \rightarrow$ $\tau^{-} \tau^{+}$is an essentially irreducible background to Higgs production by the reaction (1). Because the mass of $h$ is relatively close to the mass of the $Z$ boson, an appropriate cut on the tau-pair invariant mass $M_{\tau \tau}>M_{\text {cut }}$ suppresses the photon contribution, but not the contributions from $Z$ and the $Z \gamma$ interference term to the squared Drell-Yan matrix element.

Because our method of determining the CP nature of $h$ uses the distribution (14) in the $a^{-} a^{\prime+} \mathrm{ZMF}$, we need the corresponding distribution for Drell-Yan production of $\tau$ pairs. The spin correlations of the $\tau$ pairs produced by the intermediate vector bosons and the subsequent angular correlations between $a^{-}$and $a^{\prime+}$ differ from the correlations (13) induced 
by $h$ decay. In particular, unlike in $h$ decay, ${ }^{1}$ the $\tau^{\mp}$ samples are longitudinally polarized to some degree due to the parityviolating couplings of the $Z$ boson. In order to exhibit these features we consider Drell-Yan production of $\tau$ pairs and their subsequent decays to charged prongs $a^{-}$and $a^{\prime+}$ to lowest order in the SM couplings. The corresponding parton reaction is

$q+\bar{q} \rightarrow \gamma^{*}, Z^{*} \rightarrow \tau^{-}+\tau^{+} \rightarrow a^{-}+a^{\prime+}+X$.

For the partonic differential cross section, which is analogous to (13), we obtain ${ }^{2}$ with (11), neglecting terms of order $m_{\tau} / \sqrt{s}$ :

$$
\begin{aligned}
d \hat{\sigma}_{D Y}^{(0)}= & \frac{1}{576 \pi^{3}} \mathrm{Br}_{\tau^{-} \rightarrow a^{-}} \mathrm{Br}_{\tau^{+} \rightarrow a^{+}+} d \cos \theta_{-} \\
& \times d \cos \theta_{+} d \phi_{-} d \phi_{+} d E_{-} d E_{+} F\left(E_{i}, \theta_{i}, \phi_{i}\right),
\end{aligned}
$$

where $i= \pm$ and

$$
\begin{aligned}
F= & n\left(E_{-}\right) n\left(E_{+}\right) \sum_{B_{1}, B_{2}=Z, \gamma} a\left(B_{1}, B_{2}\right) \\
\times & \left\{v _ { \tau } ^ { B _ { 1 } } v _ { \tau } ^ { B _ { 2 } } \left[1-b\left(E_{+}\right) b\left(E_{-}\right)\left(\cos \theta_{+} \cos \theta_{-}\right.\right.\right. \\
& \left.\left.+\frac{1}{2} \sin \theta_{+} \sin \theta_{-} \cos \left(\phi_{+}+\phi_{-}\right)\right)\right] \\
+ & a_{\tau}^{B_{1}} a_{\tau}^{B_{2}}\left[1-b\left(E_{+}\right) b\left(E_{-}\right)\left(\cos \theta_{+} \cos \theta_{-}\right.\right. \\
& \left.\left.\quad-\frac{1}{2} \sin \theta_{+} \sin \theta_{-} \cos \left(\phi_{+}+\phi_{-}\right)\right)\right] \\
+ & \left.\left(a_{\tau}^{B_{1}} v_{\tau}^{B_{2}}+a_{\tau}^{B_{2}} v_{\tau}^{B_{1}}\right)\left(b\left(E_{+}\right) \cos \theta_{+}-b\left(E_{-}\right) \cos \theta_{-}\right)\right\} .
\end{aligned}
$$

The angles $\theta_{ \pm}, \phi_{ \pm}$are the polar and azimuthal angles of the $a^{-}$and $a^{\prime+}$ in the $\tau \tau$ ZMF, where the direction of the $\tau^{-}$ momentum is chosen to be the $z$-axis, and the momentum of the initial quark is located in the $x, z$-plane. Furthermore,

$v_{f}^{\gamma}=Q_{f} e \quad(e>0), \quad a_{f}^{\gamma}=0$,

$v_{f}^{Z}=e \frac{T_{3 f}-2 Q_{f} s_{\theta_{W}}^{2}}{2 s_{\theta_{W}} c_{\theta_{W}}}, \quad a_{f}^{Z}=e \frac{T_{3 f}}{2 s_{\theta_{W}} c_{\theta_{W}}}$

and

$$
\begin{gathered}
a\left(B_{1}, B_{2}\right)=s \frac{v_{q}^{B_{1}} v_{q}^{B_{2}}+a_{q}^{B_{1}} a_{q}^{B_{2}}}{D\left(B_{1}\right) D^{*}\left(B_{2}\right)}, \\
D(B)=s-m_{B}^{2}+i m_{B} \Gamma_{B} .
\end{gathered}
$$

\footnotetext{
${ }^{1}$ If one takes into account higher-order electroweak corrections in $h \rightarrow$ $\tau \tau$, longitudinal $\tau^{\mp}$ polarizations are also induced in $\tau$ pair production by $h$ decay [28], which are, however, too small to be of relevance here. ${ }^{2}$ We use the matrix elements given in [90], adapted to the reactions (20).
}

Equation (22) shows that the angular correlations, which are characteristic for the $\tau$ spin correlations induced by an intermediate spin-1 boson with vector and axial vector couplings, differ from those in (13). The last term in (22) signifies the polarization of the $\tau^{ \pm}$samples. Substituting $\phi_{-}=\varphi+\phi_{+}$ in (22), where $\varphi$ is defined in Eq. (12), and integrating (21) with respect to $\phi_{+}$from 0 to $2 \pi$, the terms proportional to $\cos \left(\phi_{+}+\phi_{-}\right)$in (22) vanish. That is, the resulting hadronic distribution $d \sigma_{D Y}^{(0)} / d E_{+} d E_{-} d \cos \theta_{+} d \cos \theta_{-} d \varphi$ is independent of $\varphi$ for any final state $a^{-} a^{\prime+}$. This is displayed, for $Z^{*} / \gamma^{*} \rightarrow \tau \tau \rightarrow \pi \pi$, in Fig. 1 by the solid red line.

We find it instructive to investigate this feature in more detail. As a result we obtain a proposal for calibrating the distribution of $\varphi$, respectively, $\varphi_{\mathrm{CP}}^{*}$ with Drell-Yan $\tau \tau$ events; see below. For definiteness, we choose the charged prongs $a^{-}, a^{+}=\pi^{-}, \pi^{+}$in the following discussion. Equation (22) shows that the $Z^{*} / \gamma^{*}$ contribution to the $\varphi$ distribution is flat only if (22) is integrated over the full $2 \pi$ range of $\phi_{+}$(or alternatively of $\phi_{-}$). The $\varphi$ distribution will deviate from a flat line if the phase space of one of the pions is restricted. For instance, if one demands the $\pi^{-}$momentum to lie in the plane defined by $\phi_{-}=0$, the contribution of, for instance, the pure photon exchange to the $\varphi$ distribution (which follows from the first line in the curly bracket of Eq. (22)) is proportional to $1-c \cos \varphi$. This distribution and, therefore, the distribution of $\varphi_{\mathrm{CP}}^{*}$ differs from a flat line.

How can this be probed experimentally? We define a variable $\cos \tilde{\alpha}_{-}$by

$\cos \tilde{\alpha}_{-}=\left|\frac{\hat{\mathbf{e}}_{\mathbf{z}} \times \hat{\mathbf{k}}_{L-}}{\left|\hat{\mathbf{e}}_{\mathbf{z}} \times \hat{\mathbf{k}}_{L-}\right|} \cdot \frac{\hat{\mathbf{p}}_{L-} \times \hat{\mathbf{k}}_{L-}}{\left|\hat{\mathbf{p}}_{L-} \times \hat{\mathbf{k}}_{L-}\right|}\right|$,

which allows one to classify the $Z^{*} / \gamma^{*} \rightarrow \tau^{-} \tau^{+} \rightarrow \pi^{-} \pi^{+}$ events into events where the $\pi^{-}$is 'nearly coplanar' and 'nearly perpendicular' to the $q \tau$ production plane in the laboratory frame. In (26) the unit vectors $\hat{\mathbf{k}}_{L-}$ and $\hat{\mathbf{p}}_{L-}$ are the $\tau^{-}$and $\pi^{-}$directions of flight in the laboratory frame and $\hat{\mathbf{e}}_{z}$ points along the direction of one of the proton beams. The range of $\tilde{\alpha}_{-}$is $0 \leq \tilde{\alpha}_{-} \leq \pi / 2$. Events with $\pi^{-}$being 'nearly coplanar' ('nearly perpendicular') are defined by demanding $\tilde{\alpha}_{-}<\pi / 4\left(\tilde{\alpha}_{-}>\pi / 4\right)$. In order to define a discriminating variable in terms of measurable quantities, we use the impact parameter vector $\hat{\mathbf{n}}_{-}$(cf. Sect. 4) instead of $\hat{\mathbf{k}}_{L-}$ and replace (26) by

$\cos \alpha_{-}=\left|\frac{\hat{\mathbf{e}_{\mathbf{z}}} \times \hat{\mathbf{p}}_{L-}}{\mid \hat{\hat{\mathbf{z}}_{\mathbf{z}} \times \hat{\mathbf{p}}_{L-} \mid}} \cdot \frac{\hat{\mathbf{n}}_{-} \times \hat{\mathbf{p}}_{L-}}{\left|\hat{\mathbf{n}}_{-} \times \hat{\mathbf{p}}_{L-}\right|}\right|$.

This variable is nearly identical to (26), i.e., events with $\pi^{-}$ being 'nearly coplanar' ('nearly perpendicular') are in the following defined by requiring $\alpha_{-}<\pi / 4\left(\alpha_{-}>\pi / 4\right)$. 


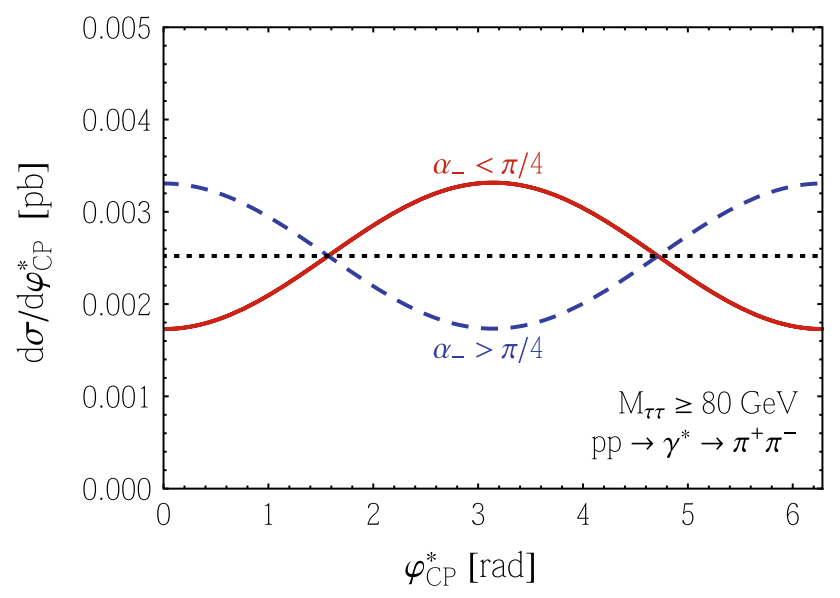

Fig. 2 Drell-Yan production of $\tau^{-} \tau^{+}$and subsequent decays to pions via photon exchange, $p p \rightarrow \gamma^{*} \rightarrow \tau^{-} \tau^{+} \rightarrow \pi^{+} \pi^{-}+2 \nu$. Left: The unnormalized distribution of $\varphi_{\mathrm{CP}}^{*}$ for events with $\pi^{-}$'nearly coplanar' $\left(\alpha_{-}<\pi / 4\right.$, red solid line $)$ and events with $\pi^{-}$'nearly perpendicular' $\left(\alpha_{-}>\pi / 4\right.$, dashed blue line $)$ to the $q \tau$ production plane. The

Let us first consider Drell-Yan production of $\tau^{-} \tau^{+}$and subsequent decays to pions via photon exchange, $p p \rightarrow$ $\gamma^{*} \rightarrow \tau^{-} \tau^{+} \rightarrow \pi^{+} \pi^{-}+2 v$. The $\varphi_{\mathrm{CP}}^{*}$ distributions [computed with the first line in the curly bracket of (22)] are shown in Fig. 2, left plot, for events with $\pi^{-}$'nearly coplanar' $\left(\alpha_{-}<\pi / 4\right.$, red solid line) and events with $\pi^{-}$'nearly perpendicular' ( $\alpha_{-}>\pi / 4$, dashed blue line) to the $q \tau$ production plane.

The distribution for events with $\pi^{-}$'nearly coplanar' is enhanced for $\varphi_{\mathrm{CP}}^{*} \sim \pi$ which corresponds to $\pi^{-}$and $\pi^{+}$ being (nearly) antiparallel. In order to understand this let us consider $q \bar{q} \rightarrow \gamma^{*} \rightarrow \tau^{-} \tau^{+}$where, for illustration, the $\tau$ pair is emitted perpendicular to the incoming quark direction. The $\tau$ pair is produced in a s-wave and the resulting spin configuration for this type of events is shown in the right plot of Fig. 2, i.e., the $\tau^{-}$and $\tau^{+}$spin projections onto the quark axis are parallel. The $\tau^{ \pm}$decay distributions (11) tell us that the $\pi^{-}\left(\pi^{+}\right)$are then preferentially emitted in (opposite to) the direction of the $\tau^{-}\left(\tau^{+}\right)$spin, which means that the $\varphi_{\mathrm{CP}}^{*}$ distribution is enhanced for $\phi_{-}-\phi_{+} \sim \pi$.

On the other hand if the $\pi^{-}$is emitted 'nearly perpendicular' to the $q \tau$ production plane $\left(\alpha_{-}>\pi / 4\right)$, the $\varphi_{\mathrm{CP}}^{*}$ distribution is enhanced at $\varphi_{\mathrm{CP}}^{*} \sim 0$ and $2 \pi$, cf. the left plot of Fig. 2. Again this can be understood from the right plot of Fig. 2 and the $\tau^{ \pm}$decay distributions (11). The projection of the spin of $\gamma^{*}$ and thus the projection of the total $\tau^{-} \tau^{+}$ spin onto the axis orthogonal to the $q \tau$ production plane is zero, i.e., the $\tau^{-}$and $\tau^{+}$spins are predominantly anticorrelated with respect to this axis ('up-down' and 'downup'). Therefore, the momenta of the $\pi^{-}$and $\pi^{+}$are preferentially parallel in this case. The $\varphi_{\mathrm{CP}}^{*}$ distributions for the two sets of events $\left(\alpha_{-}<\pi / 4\right.$ and $\left.\alpha_{-}>\pi / 4\right)$ add up

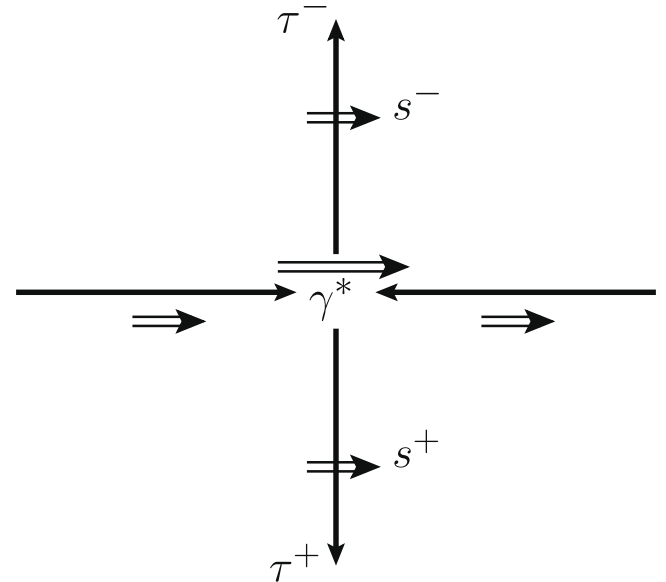

dotted black line is half of the sum of the two distributions. The cuts $M_{\tau \tau} \geq 80 \mathrm{GeV}$ and $\left|\eta_{\pi^{ \pm}}\right| \leq 1$ were used. Right: Spin configuration for $q \bar{q} \rightarrow \gamma^{*} \rightarrow \tau^{-} \tau^{+}$events where the $\tau^{-} \tau^{+}$are produced orthogonal to the beam direction

exactly to a flat line as stated above and already shown in Fig. 1.

Let us now consider (20) with the intermediate $Z$ boson. In view of the analysis of Sect. 6 below, we apply a cut on the $\tau$-pair invariant mass $M_{\tau \tau} \geq M_{\text {cut }}\left(M_{\text {cut }} \gtrsim 80 \mathrm{GeV}\right)$. Then (20) is dominated by $Z$-boson exchange. The strengths of the vector and axial vector couplings of the $\tau$ leptons imply that the differential cross section (21) is dominated by the second line of the curly bracket in (22), i.e., by $\tau$-pair production through the axial vector current, which corresponds to $\mathrm{p}$ wave production of $\tau^{-} \tau^{+}$. The resulting $\tau$ spin correlations differ from those induced by $\gamma^{*}$ exchange discussed above. In the case of axial vector production the $\tau^{-}$and $\tau^{+}$spin projections onto the quark axis are predominantly anticorrelated (excluding the forward and backward regions), while the $\tau^{-}$ and $\tau^{+}$spin projections onto the axis orthogonal to the $q \tau$ production plane are predominantly correlated. Therefore, in the case of axial vector production, the $\varphi_{\mathrm{CP}}^{*}$ distributions for events with $\pi^{-}$emitted 'nearly coplanar' and 'nearly perpendicular', respectively, are opposite to the corresponding distributions for $\gamma^{*}$ exchange shown in Fig. 2.

The left plot of Fig. 3 shows the corresponding unnormalized $\varphi_{\mathrm{CP}}^{*}$ distributions computed with the complete tree-level differential cross section (21), (22). The cuts $M_{\tau \tau} \geq 80 \mathrm{GeV}$ and $\left|\eta_{\pi^{ \pm}}\right| \leq 1$ on the pseudo-rapidity of the pions were applied. The shape of these distributions reflect the outcome of the discussion made in the previous paragraph. ${ }^{3}$ Again, the two distributions add up to a flat line.

\footnotetext{
${ }^{3}$ As to the signal reaction $p p \rightarrow h \rightarrow \tau^{-} \tau^{+} \rightarrow \pi^{-} \pi^{+} 2 \nu$ we remark the following. For a Higgs boson of any CP nature, the unnormalized $\varphi_{\mathrm{CP}}^{*}$ distributions have the same shape for events with $\pi^{-}$being nearly coplanar and nearly perpendicular.
} 


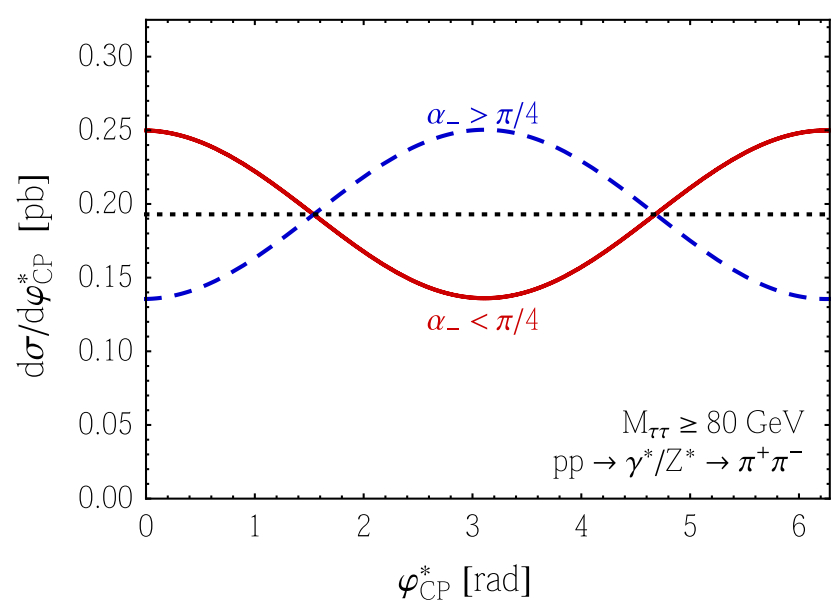

Fig. 3 Drell-Yan production of $\tau^{-} \tau^{+}$and subsequent decays to pions via $Z^{*} / \gamma^{*}$ exchange, $p p \rightarrow Z^{*} / \gamma^{*} \rightarrow \tau^{-} \tau^{+} \rightarrow \pi^{+} \pi^{-}+2 v$. The unnormalized distribution of $\varphi_{\mathrm{CP}}^{*}$ for events with $\pi^{-}$'nearly coplanar' $\left(\alpha_{-}<\pi / 4\right.$, red solid line) and events with $\pi^{-}$'nearly perpendicu-

Contrary to the case of an intermediate Higgs boson, the tree-level distributions (22) will be affected by higher-order QCD corrections to (20) because of the correlations of the $\tau$ spins with the initial-state parton momenta. We have computed the respective differential distributions for

$p p \rightarrow Z^{*}, \gamma^{*}+X \rightarrow \tau^{-}+\tau^{+}+X \rightarrow a^{-}+a^{\prime+}+X$

at next-to-leading order (NLO) in the QCD coupling $\alpha_{s}$, taking the $\tau$ spin correlations in the virtual and real corrections into account. We calculated the respective $\tau$ spin density matrices for $q \bar{q} \rightarrow Z^{*}, \gamma^{*}(g) \rightarrow \tau^{-} \tau^{+}(g)$ and $g q(\bar{q}) \rightarrow Z^{*}, \gamma^{*} q(\bar{q}) \rightarrow \tau^{-} \tau^{+} q(\bar{q})$ at order $\alpha_{s}$. The soft and collinear divergences are treated with Catani-Seymour dipole subtraction [91] with collinear factorization in the $\overline{\mathrm{MS}}$ scheme.

As to the NLO QCD $\varphi_{\mathrm{CP}}^{*}$ distributions for $Z^{*} / \gamma^{*} \rightarrow$ $\tau^{-} \tau^{+} \rightarrow \pi^{-} \pi^{+}+X$ for events with $\alpha_{-}<\pi / 4$ and $\alpha_{-}>\pi / 4$ : they are displayed in the right plot Fig. 3. The comparison with the LO distributions shows that the order $\alpha_{s}$ QCD corrections amount to about $12 \%$ and the shapes of these NLO distributions remain essentially the same as the LO distributions.

The results shown in Fig. 3 suggest that one may use the Drell-Yan events $p p \rightarrow Z^{*} / \gamma^{*} \rightarrow \tau \tau \rightarrow \pi \pi \nu \bar{\nu}$, which are quite abundant at the LHC, for experimentally calibrating and validating the $\varphi_{\mathrm{CP}}^{*}$ distribution(s) before this observable is used to determine the CP nature of the Higgs boson $h$.

The NLO QCD polar angle and energy distributions of the charged prongs $a a^{\prime}$, which can be used for background suppression, will be discussed in the next section.

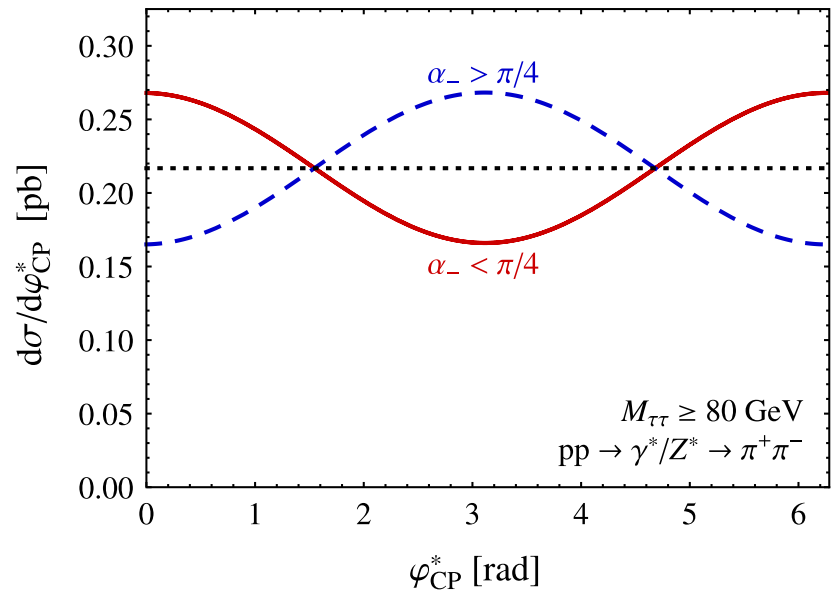

lar' $\left(\alpha_{-}>\pi / 4\right.$, dashed blue line $)$ to the $q \tau$ production plane. The dotted black line is half of the sum of the two distributions. The cuts $M_{\tau \tau} \geq 80 \mathrm{GeV}$ and $\left|\eta_{\pi^{ \pm}}\right| \leq 1$ were used. Left plot: LO QCD. Right plot: NLO QCD

\section{Numerical results for the $\mathrm{LHC}$}

In this section we consider Higgs production (1) and decay into $\tau$ pairs (2) at the LHC for a collider center-of-mass energy $\sqrt{S}=14 \mathrm{TeV}$. We analyze how the differences between the Higgs-boson induced and $Z^{*} / \gamma^{*}$ induced $\tau$ spin correlations, respectively the differences between the resulting $a a^{\prime}$ angular and energy correlations can be used to reduce the $Z^{*} / \gamma^{*}$ background. At the end of this section we estimate the precision $\Delta \phi_{\tau}$ with which the Higgs mixing angle may be determined in the $h \rightarrow \tau \tau$ decay mode at the LHC.

As long as no cuts on the final-state particles/jets are applied, the normalized distributions (13) for the signal reaction (1) hold also when higher-order QCD corrections are taken into account. In order to obtain NLO QCD distributions with cuts for (1), we compute the two-dimensional distributions of the Higgs-boson transverse momentum and rapidity by means of the computer code MCFM $[81,82,92,93]$. We include $h$ decay into $\tau$ pairs with $\tau$ spin correlations and their subsequent decays into charged prongs by Monte Carlo simulation. We put $m_{h}=125 \mathrm{GeV}$ and assume the $g g \rightarrow h$ amplitude to be the same as in the SM, and we take $\Gamma_{h}=\Gamma_{h}^{S M}=4.29 \mathrm{MeV}$. For estimating signal-tobackground ratios we take the $\mathrm{K}$ factor for inclusive Higgs production, $\mathrm{K}_{\mathrm{NNLO} / \mathrm{NLO}}=1.35$ into account, calculated with the computer code HNNLO [94-96]. We calculated the angular distributions analogous to (21) and corresponding energy distributions for the background reactions (28) also to NLO QCD (cf. above). For the numerical evaluation we used the parton distribution functions CT10 [97] with $\alpha_{s}\left(m_{Z}\right)=0.1180$ and two-loop running in $\alpha_{s}$. Moreover, we used $\alpha\left(M_{Z}\right)=1 / 128.89$ and the weak mixing angle 

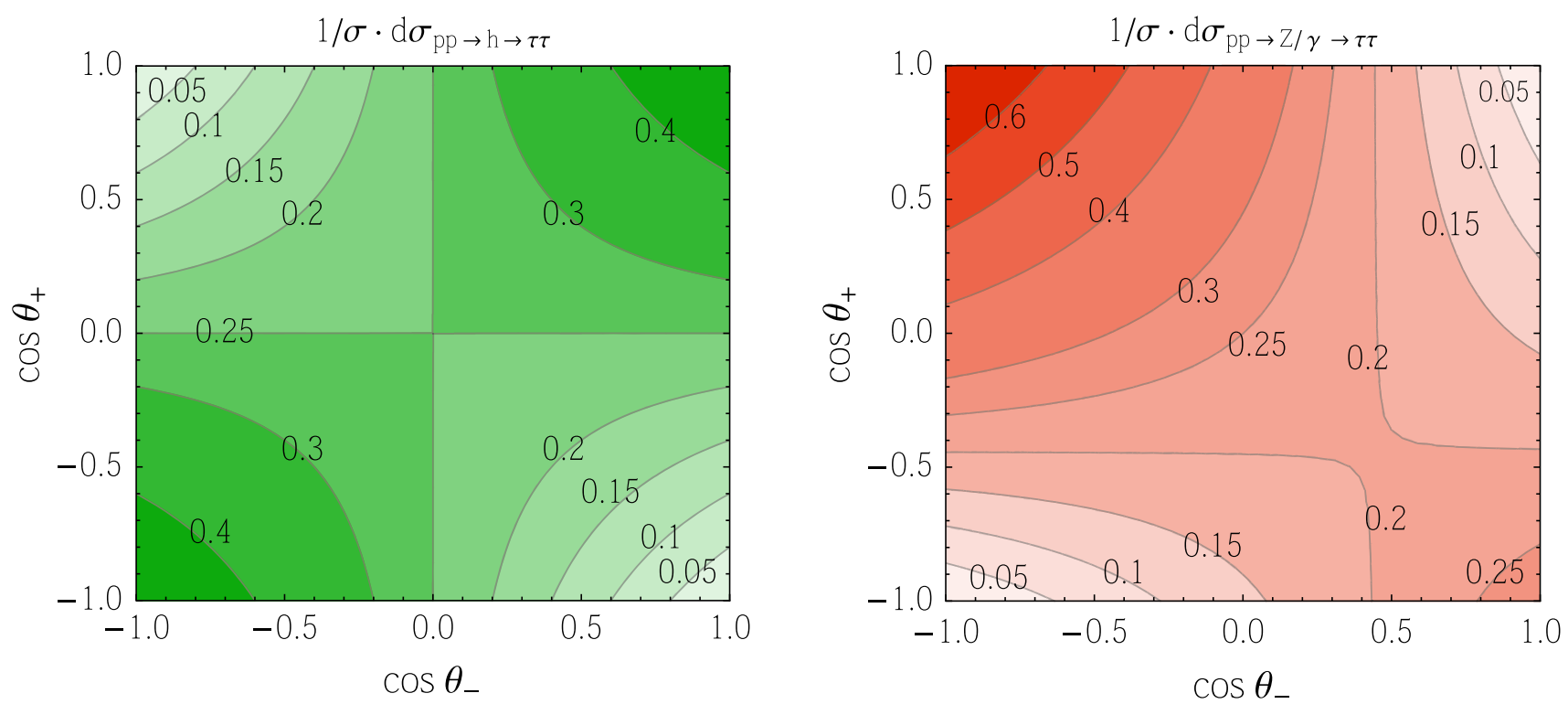

Fig. 4 LHC, $\sqrt{S}=14 \mathrm{TeV}, M_{\tau \tau} \geq 100 \mathrm{GeV}$. Production of $h+X$ (left) of arbitrary CP nature and of $Z^{*} / \gamma^{*}+X$ (right) with subsequent decay of the respective boson to $\tau^{-} \tau^{+} \rightarrow \pi^{+} \pi^{-}+2 \nu$. The plots show the distributions $\sigma^{-1} d \sigma / d \cos \theta_{-} d \cos \theta_{+}$without cuts on the pion momenta

$\sin ^{2} \theta_{W}=0.2228$. As a default value for the renormalization and factorization scale $\mu$ we have set $\mu=\mu_{R}=\mu_{F}=$ $m_{h}$.

The ATLAS and CMS experiments, which reported evidence for $h \rightarrow \tau \tau[8,10]$, have reconstructed the $\tau \tau$ invariant mass with methods described in [98] and [10], respectively. The Higgs-boson signal appears as an enhancement of $d \sigma / d M_{\tau \tau}$ in a mass window around $M_{\tau \tau}=m_{h}$ over the background which is mostly due to $Z^{*} / \gamma^{*} \rightarrow \tau \tau$. If not stated otherwise, we apply in the following sections a cut $M_{\tau \tau}>100 \mathrm{GeV}$, which strongly suppresses the background from $Z^{*} / \gamma^{*} \rightarrow \tau \tau$.

\section{$6.1 \tau^{+} \tau^{-}$spin correlations and subsequent polar angle and energy correlations}

We analyze the distributions $d \sigma / d \cos \theta_{-} d \cos \theta_{+}$and associated energy distributions for the signal and background reactions, where the helicity angles $\theta_{\mp}$ of $a^{-}$and $a^{\prime+}$ are defined as in Sect. 3. In the left and right plot of Fig. 4 the normalized distributions $\sigma^{-1} d \sigma / d \cos \theta_{-} d \cos \theta_{+}$at NLO QCD are shown for the signal and background reaction for the $\tau^{-} \tau^{+} \rightarrow \pi^{-} \pi^{+}+2 v$ decay mode. No cuts on the pion transverse momentum $p_{T}^{\pi}$ or the pion rapidity are applied. Solid gray contour lines denote constant values. The normalized signal distribution is given by $\left(1+\cos \theta_{-} \cos \theta_{+}\right) / 4$, cf. Eq. (13), and becomes maximal for $\cos \theta_{-} \cos \theta_{+} \rightarrow 1$. The background distribution contains terms proportional to $1-\cos \theta_{-} \cos \theta_{+}$due to the $Z^{*} / \gamma^{*}$ induced $\tau$ spin correlations and, in addition, terms linear in $\cos \theta_{\mp}$ which sig- nify a longitudinal $\tau^{\mp}$ polarization generated by the parityviolating $Z \tau \tau$ coupling. The background can be reduced by applying a cut on the product of $\operatorname{cosines,} \cos \theta_{-} \cos \theta_{+}>$ $-c_{\min }\left(c_{\min }>0\right)$, where $c_{\min }$ should be different for $\cos \theta_{-} \rightarrow \pm 1$, in view of the asymmetric background distribution. These cuts should not be too hard because that part of the signal cross section which is sensitive to the Higgs $C P$ mixing angle $\phi_{\tau}$ is proportional to $\sin \theta_{-} \sin \theta_{+}$, cf. Eq. (13).

The uncertainties due to scale variations of these NLO distributions are as follows. The normalized signal distribution in Fig. 4, left, is identical to the normalized LO distribution because production and decay of the Higgs boson factorizes. In order to estimate the scale uncertainty of the normalized $Z^{*} / \gamma^{*}$ distribution in Fig. 4, right, we vary the scale $\mu$ between $\mu=m_{h} / 2$ and $\mu=2 m_{h}$ and calculate the deviation $\left[\sigma^{-1} d \sigma\left(\mu=m_{h}\right)-\sigma^{-1} d \sigma(\mu)\right] / \sigma^{-1} d \sigma\left(\mu=m_{h}\right)$ for each value of $\cos \theta_{-}$and $\cos \theta_{+}$. Apart from a small region in the lower left and upper right corner of Fig. 4, right, the maximal deviation of each point from the respective value of $1 / \sigma \cdot d \sigma\left(\mu=m_{h}\right)$ is small, about $\pm 3 \%$.

Both distributions in Fig. 4 are affected if a $p_{T}$ cut on the pion momenta is applied. A $p_{T}^{\pi}$ cut mostly removes events in the vicinity of $\cos \theta_{-}=-1$ and $\cos \theta_{+}=+1$, because in these phase-space regions the pion is emitted opposite to the corresponding $\tau$ direction of flight and, therefore, its energy in the $\tau \tau \mathrm{ZMF}$ is small.

Cuts on $\cos \theta_{\mp}$ may be unrealistic because, at the LHC, the reconstruction of the $\tau$ rest frames is complicated, even for hadronic $\tau$ decays. This is because the partonic center- 

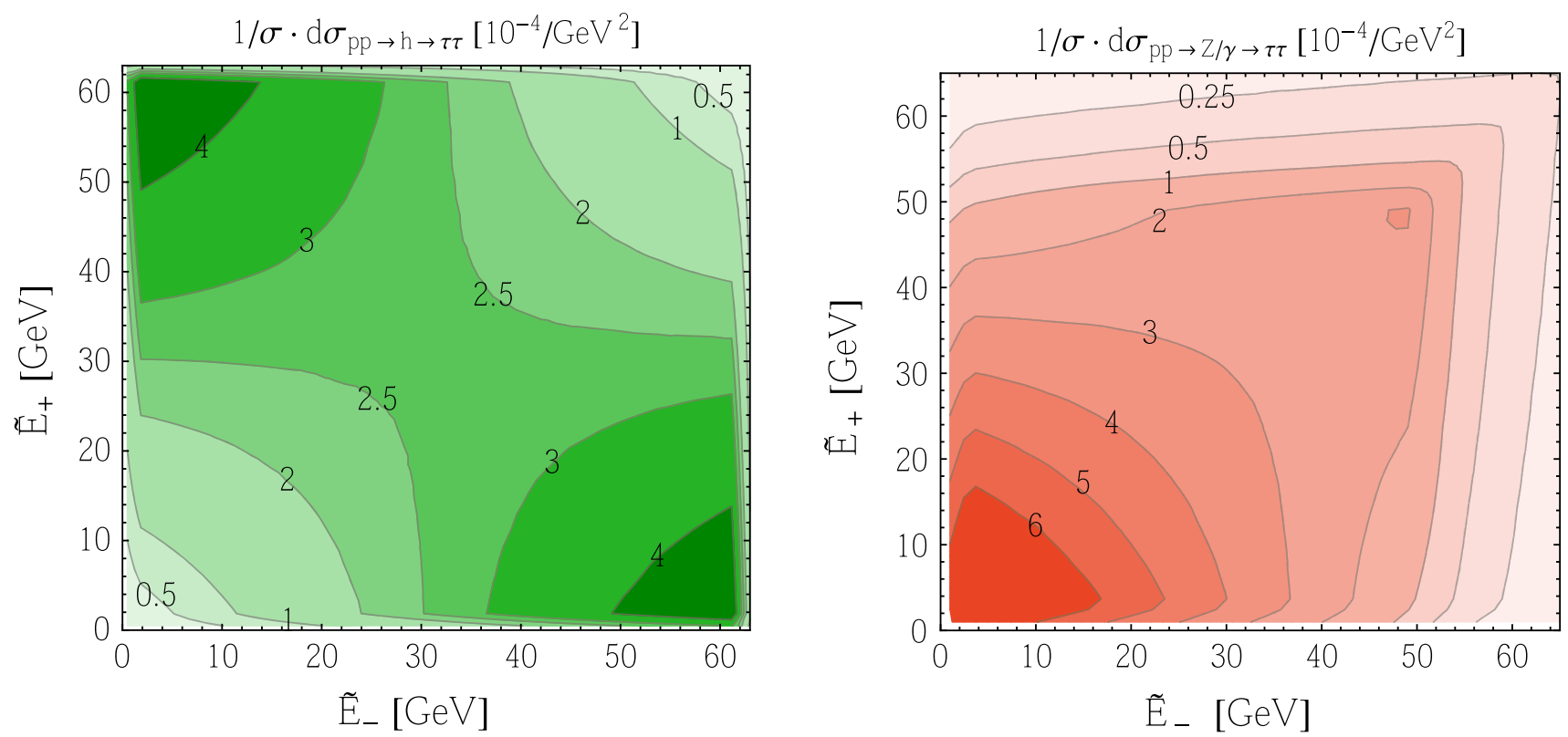

Fig. $5 \mathrm{LHC}, \sqrt{S}=14 \mathrm{TeV}, M_{\tau \tau} \geq 100 \mathrm{GeV}$. Production of $h+X$ (left) of arbitrary CP nature and of $Z^{*} / \gamma^{*}+X$ (right) with subsequent decay of the respective boson to $\tau^{-} \tau^{+} \rightarrow \pi^{+} \pi^{-}+2 \nu$. The plots show the distributions $\sigma^{-1} d \sigma / d \tilde{E}_{-} d \tilde{E}_{+}$without cuts on the pion momenta

of-mass energy is unknown for a certain event and the decay of each $\tau \tau$ pair involves at least two neutrinos. However, one can approximately reconstruct the $\tau \tau$ ZMF with a fitting procedure [98] and determine the pion energies in this frame, denoted by $\tilde{E}_{\mp}$ in the following. The energies $\tilde{E}_{\mp}$ are related to $\cos \theta_{\mp}$ by boosts. In Fig. 5 the NLO QCD distributions $\sigma^{-1} d \sigma / d \tilde{E}_{-} d \tilde{E}_{+}$are displayed for the signal and background reaction. Solid gray contour lines denote constant values. The normalized signal distribution in Fig. 5, left, shows that $h \rightarrow \tau \tau$ events decay preferably into one pion with a large energy and one pion with a small energy in the $\tau \tau$ ZMF. On the other hand, the right plot of Fig. 5 shows that in the case of the $Z^{*} / \gamma^{*} \rightarrow \tau \tau$ background, events where both pion energies are small are strongly enhanced.

If one applies a cut on the transverse momenta of the $\pi^{\mp}$, for instance, $p_{T}^{\pi} \geq 20 \mathrm{GeV}$, the number of events with small $\tilde{E}_{\mp}$ are reduced. At LO QCD this cut removes all events with $\tilde{E}_{\mp}<20 \mathrm{GeV}$ because the transverse momentum of the $h, Z^{*}, \gamma^{*}$ boson is zero. At NLO QCD this is lifted to some extent because the finite transverse momentum of the respective boson results in $p_{T}^{\pi} \gtrsim 20 \mathrm{GeV}$ even if $\tilde{E}_{\mp}<$ $20 \mathrm{GeV}$ in the $\tau \tau \mathrm{ZMF}$.

The normalized distributions of Fig. 5 suggest the application of cuts on the energies $\tilde{E}_{\mp}$ in order to enhance the signal-to-background ratio. This ratio is enhanced by rejecting events where both $\tilde{E}_{+}$and $\tilde{E}_{-}$are smaller than, for instance, $20 \mathrm{GeV}$, or where both energies are larger than $45 \mathrm{GeV}$. However, one should not reject regions including $\tilde{E}_{+} \sim \tilde{E}_{+} \sim 30 \mathrm{GeV}$ which corresponds to $\sin \theta_{-} \sin \theta_{+} \sim$
1. Here the sensitivity to $\varphi_{\mathrm{CP}}^{*}$ and therefore to the mixing angle $\phi_{\tau}$ is largest [cf. Eq. (13)].

6.2 The distribution of $\varphi_{\mathrm{CP}}^{*}$ for $h \rightarrow \tau \tau$

\subsubsection{Direct $\tau^{+} \tau^{-} \rightarrow \pi^{+}+\pi^{-}+2 v$ decay}

The normalized $\varphi_{\mathrm{CP}}^{*}$ distribution without cuts is shown in Fig. 1 for $h \rightarrow \tau^{+} \tau^{-} \rightarrow \pi^{+} \pi^{-}+2 v$. If no cuts on the final-state particles are applied, the distribution is the same also for other Higgs production modes, or if higher-order QCD corrections are included. The distribution will change if kinematical cuts like $p_{T}^{\pi}$ cuts are applied, because the $\varphi$ distribution results from the term proportional to $\sin \theta_{+} \sin \theta_{-}$in Eq. (13). Enhancing the region $\cos \theta_{+} \cos \theta_{-} \sim 0$ of Fig. 4, left, increases the asymmetry defined in Eq. (18). The dependence of the normalized $\varphi_{\mathrm{CP}}^{*}$ distribution at NLO QCD on cuts on $p_{T}^{\pi}$ and on $\tilde{E}_{\mp}$ is displayed in Fig. 6, left, for a Higgs mixing angle $\phi_{\tau}=-\pi / 4$. The solid black line shows the distribution without cuts. The corresponding asymmetry is $A^{\pi \pi}=39.3 \%$. If a cut $p_{T}^{\pi} \geq 20 \mathrm{GeV}$ is applied the asymmetry, associated with the distribution shown by the dashed black line, increases to $A_{\mathrm{NLO}}^{\pi \pi}=49.5 \%\left(A_{\mathrm{LO}}^{\pi \pi}=50.2 \%\right)$. For the Higgs-boson production mode (1) the Higgs-boson transverse momentum is, on average, small. Therefore the $p_{T}^{\pi}$ cut removes events with $E_{ \pm}^{\tau \tau} \lesssim 20 \mathrm{GeV}$ in the distribution displayed in Fig. 5, left. For these events the value of $\sin \theta_{ \pm}$ is small for at least one of the pions. Therefore the value of the product $\sin \theta_{+} \sin \theta_{-}$is on average rather large for the remaining events. This is why the asymmetry $A$ is increased 


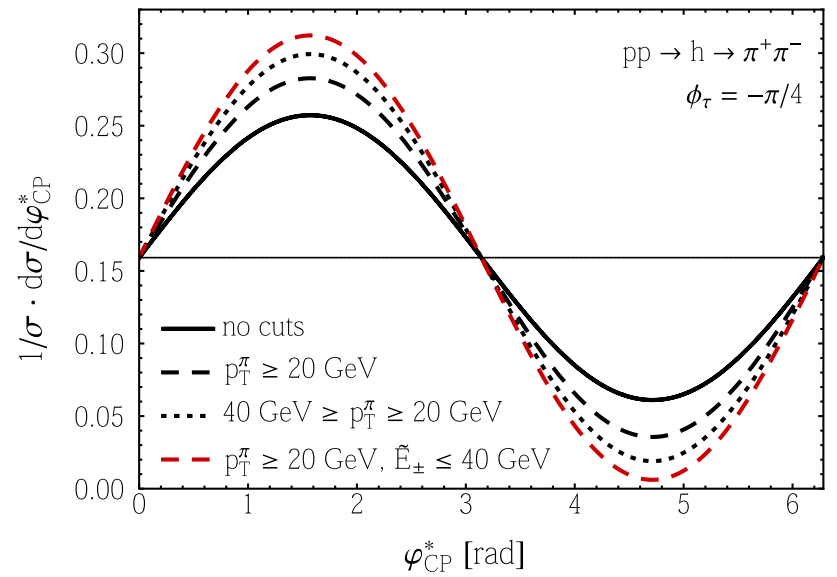

Fig. 6 Left: $p p \rightarrow h \rightarrow \tau^{-} \tau^{+} \rightarrow \pi^{+} \pi^{-}+2 \nu$, where $h$ is assumed to be a $\mathrm{CP}$ mixture with mixing angle $\phi_{\tau}=-\pi / 4$. Dependence of the distribution $\sigma^{-1} d \sigma / d \varphi_{\mathrm{CP}}^{*}$ on different kinematical cuts. Right:

by this cut. If, in addition, events with large transverse pion momenta are rejected by selecting, for instance, events with $40 \mathrm{GeV} \geq p_{T}^{\pi} \geq 20 \mathrm{GeV}$, the asymmetry is further enhanced to $56 \%\left(A_{\mathrm{LO}}^{\pi \pi}=59.5 \%\right)$. The corresponding $\varphi_{\mathrm{CP}}^{*}$ distribution is given by the dotted black line in Fig. 6. Cuts on the pion energies will also change this distribution and the resulting asymmetry. The $\varphi_{\mathrm{CP}}^{*}$ distribution shown by dashed red line in Fig. 6, left, results from applying the cuts $p_{T}^{\pi} \geq 20 \mathrm{GeV}$ and $\tilde{E}_{ \pm} \leq 40 \mathrm{GeV}$. The corresponding asymmetry is $61.2 \%$ $\left(A_{\mathrm{LO}}^{\pi \pi}=61.8 \%\right)$.

Notice that all these cuts do not change the location of the maximum of the $\varphi_{\mathrm{CP}}^{*}$ distribution. Furthermore, cuts on the pseudo-rapidity of the pions $\eta_{\pi}$ do not change the normalized $\varphi_{\mathrm{CP}}^{*}$ distributions displayed in Fig. 6.

\subsubsection{Other $\tau$ decay modes}

At the end of Sect. 6.1, cuts on the energies $\tilde{E}_{\mp}$ of the charged pions were suggested for the direct $\tau \rightarrow \pi$ decays, in order to enhance the signal-to-background ratio and the asymmetry (18). If the Higgs mixing angle $\phi_{\tau}$ is to be determined from other $\tau$ decay modes with the method described in Sect. 4, e.g. from $\tau^{+} \tau^{-} \rightarrow \rho^{+} \rho^{-}+2 v$ and subsequent $\rho^{ \pm} \rightarrow \pi^{ \pm}+\pi^{0}$ decay, these cuts on $\tilde{E}_{\mp}$ can, however, not be used for background suppression in this case. This is because the $\tau$-spin analyzing power of the charged pion from $\rho$ decay is energydependent, cf. for instance Fig. 4 in [56], where this analyzing power is shown as a function of the pion energy in the $\tau$ rest frame. This energy is related by a boost to the $\pi^{ \pm}$energy $\tilde{E}_{ \pm}$ in the $\tau \tau$ ZMF. Dividing the $\tilde{E}_{-}, \tilde{E}_{+}$phase space into four regions, two with $\tilde{E}_{+} \leq 30 \mathrm{GeV}, \tilde{E}_{+} \geq 30 \mathrm{GeV}$ and two, where one energy is smaller than and the other one larger than $30 \mathrm{GeV}$, we show in Fig. 6, right, the resulting normalized distributions of the angle $\varphi_{\mathrm{CP}}^{*}$. The distribution given by the red

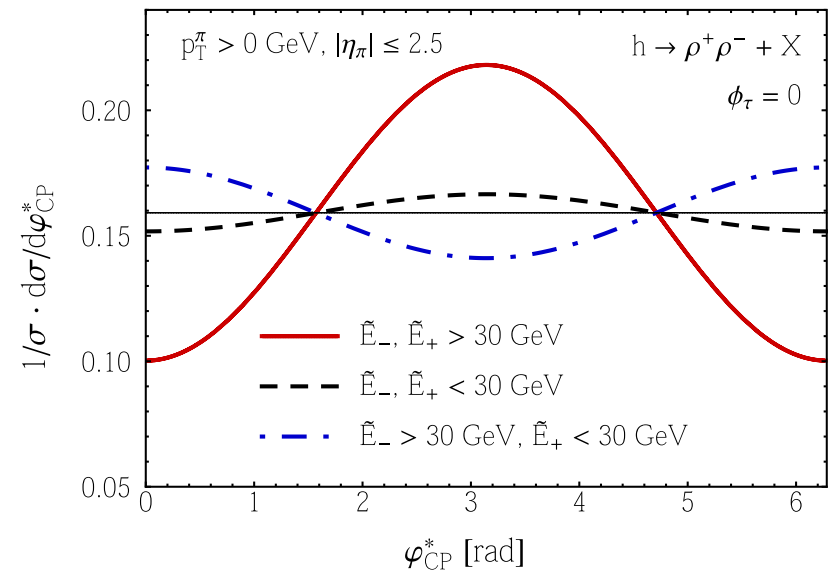

$h \rightarrow \tau^{+} \tau^{-} \rightarrow \rho^{+} \rho^{-}+2 v$ for a CP-even Higgs boson. Dependence of the distribution $\sigma^{-1} d \sigma / d \varphi_{\mathrm{CP}}^{*}$ on cuts on the energies $\tilde{E}_{\mp}$ of the charged pions. The horizontal lines in both plots are drawn to guide the eye

solid line, which has the largest asymmetry (18), results from events where both energies $\tilde{E}_{\mp} \geq 30 \mathrm{GeV}$. For events with $\tilde{E}_{\mp} \leq 30 \mathrm{GeV}$ the distribution is almost flat (dashed black line). For events with $\tilde{E}_{-} \geq 30 \mathrm{GeV}$ and $\tilde{E}_{+} \leq 30 \mathrm{GeV}$ (dotdashed blue line) the resulting asymmetry is also quite small. Furthermore, the $\varphi_{\mathrm{CP}}^{*}$ distribution is shifted in this case by an angle $\pi$ with respect to the solid red line. This is because for $\tau \rightarrow \rho \rightarrow \pi$ decay, the function $b(E)$ in (11), which encodes the $\tau$-spin analyzing power of the charged pion for this decay mode, is negative for $\tilde{E} \leq 30 \mathrm{GeV}$. The asymmetry (18) is largest for events with $\tilde{E}_{\mp} \geq 30 \mathrm{GeV}$ because if $\tilde{E}_{ \pm}$are large, the pion energies in the respective $\tau$ rest frames are also large on average. In this energy range the $\tau$-spin analyzing power of the charged pion from $\rho$ decay is large (and positive).

\subsubsection{Impact of measurement uncertainties}

The normalized $\varphi_{\mathrm{CP}}^{*}$ distributions are affected by measurement uncertainties, in particular by the uncertainties associated with the measurements of the directions $\hat{\mathbf{n}}_{\mp}$ of the impact parameters of the charged prongs $a^{-}, a^{\prime+}$ (cf. Sect. 4). In order to assess the effect of these uncertainties on the distributions $\varphi_{\mathrm{CP}}^{*}$ for the various $\tau \tau$ decay modes with Monte Carlo methods, we have "smeared" the relevant quantities with a Gaussian distribution function $\propto \exp \left(-(X / \sigma)^{2} / 2\right)$. Here $X$ denotes the generated quantity (coordinate in position space, momentum component, energy) and $\sigma$ its expected standard deviation.

The primary vertex (PV), i.e., the Higgs-boson production/decay vertex is varied along and transverse to the beam axis with $\sigma_{z}^{P V}=20 \mu \mathrm{m}$ and $\sigma_{t r}^{P V}=10 \mu \mathrm{m}$, respectively. In the following, we discuss the effect of smearing in some detail for the $\tau^{-} \tau^{+} \rightarrow \pi^{-} \pi^{+}$decay mode. The intersection point of the impact parameter vector $\mathbf{n}_{\mp}$ with the respective track 


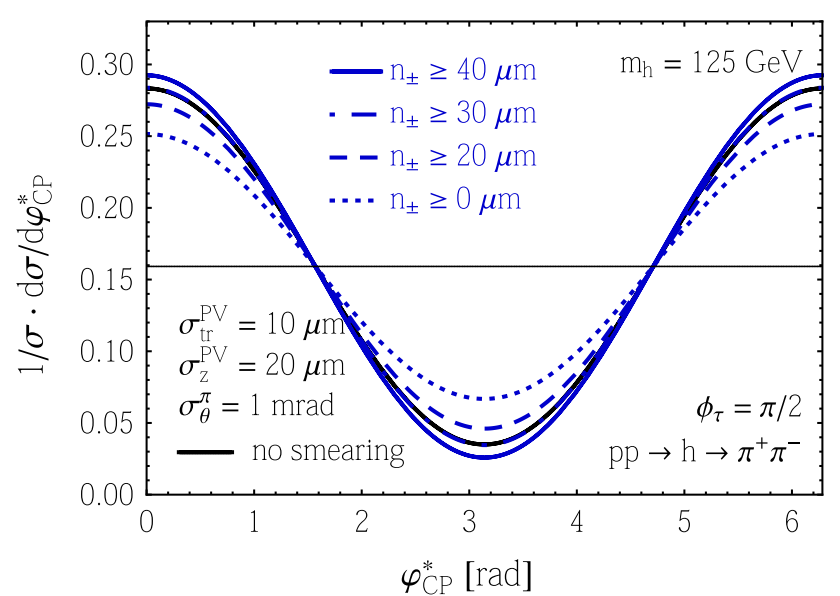

Fig. $7 \operatorname{LHC}(\sqrt{S}=14 \mathrm{TeV}), p p \rightarrow h \rightarrow \tau^{-} \tau^{+} \rightarrow \pi^{+} \pi^{-}+2 v$ with cuts $p_{T}^{\pi} \geq 20 \mathrm{GeV},\left|\eta_{\pi}\right| \leq 2.5$. The normalized $\varphi_{\mathrm{CP}}^{*}$ distribution, taking measurement uncertainties into account, for different minimum cuts on

of the charged pion $\pi^{\mp}$ is varied by $\sigma_{t r}^{\pi}=10 \mu \mathrm{m}$ within a circle transverse to the direction of the pion momentum. Furthermore we assume the angular resolution of the charged $\pi^{\mp}$ track at its intersection point with $\mathbf{n}_{\mp}$ to be distributed with $\sigma_{\theta}^{\pi}=1 \mathrm{mrad}$, and the resolution of the $\pi^{ \pm}$energy is taken to be $\Delta E^{\pi} / E^{\pi}=5 \%$. With these values, suggested in $[99,100]$, we arrive at the conclusion that a rather precise measurement of the Higgs mixing angle $\phi_{\tau}$ is possible at the LHC; see below.

First, we determine the average length $\left\langle\left|\mathbf{n}_{\mp}\right|\right\rangle$ of the impact parameter in the laboratory frame. We use an exponential decay law for the $\tau$ leptons from $h$-decay with an average $\tau$ decay length of $\left\langle c \tau_{\tau}\right\rangle=87 \mu \mathrm{m}$. If one assumes that the pion is emitted, in the $\tau$ rest fame, transversely to the $\tau$ direction of flight, then we obtain $\left\langle\left|\mathbf{n}_{\mp}\right|\right\rangle=44 \mu \mathrm{m}$. This estimate indicates also the magnitude of the resolution which must be experimentally achieved both for the primary vertex and the tracks of the pions.

Taking the smearing of the various quantities into account, with standard deviations as specified above, the resulting effects on the $\varphi_{\mathrm{CP}}^{*}$ distribution are shown in Fig. 7, left, for the $\tau \tau \rightarrow \pi \pi$ decay of a CP-odd Higgs boson and Fig. 7, right, for a CP-even Higgs boson. The black solid lines show the distributions without any smearing. The dotted blue lines include the effect of smearing using the parameters given above. The asymmetry (18) is then strongly reduced from $A_{\mathrm{NLO}}^{\pi \pi}=49.5 \%$ to $18 \%\left(A_{\mathrm{LO}}^{\pi \pi}=18.2 \%\right)$ in the case of a CP-even Higgs boson and to $37 \%\left(A_{\mathrm{LO}}^{\pi \pi}=37.4 \%\right)$ for a $\mathrm{CP}$-odd Higgs boson. These asymmetries can be enhanced by taking into account only events with impact parameter lengths $n_{ \pm}$above a certain minimum value. For the cuts $n_{ \pm} \geq 20 \mu \mathrm{m}, n_{ \pm} \geq 30 \mu \mathrm{m}$, and $n_{ \pm} \geq 40 \mu \mathrm{m}$, the resulting $\varphi_{\mathrm{CP}}^{*}$ distributions are displayed in Fig. 7. The associated asymmetry $A_{\mathrm{NLO}}^{\pi \pi}$ is $25.7,32.3,38.6 \%$ in the case of a CP-even Higgs boson and 45.2, 49.8, 53.3\% for a CP-odd

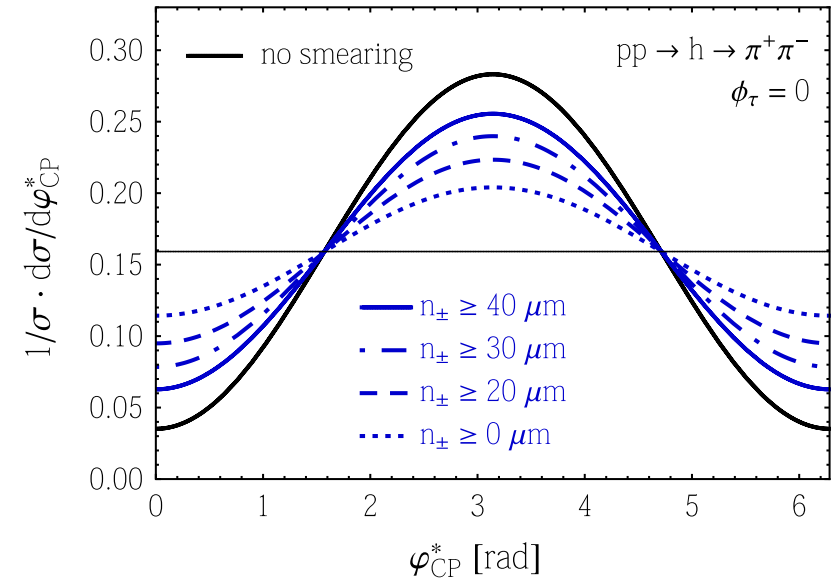

the length of the impact parameters $n_{ \pm}$. The left and right plots refer to a CP-odd and CP-even Higgs boson, respectively. The horizontal lines in both plots are drawn to guide the eye

Higgs boson. Our Monte Carlo simulations indicate that the value $n_{\min }$ of the minimum cut should be of the same size as the largest value from the set $\left\{\sigma_{z}^{P V}, \sigma_{t r}^{P V}, \sigma_{t r}^{\pi}\right\}$. Of course, the number of events is reduced by a cut on $n_{ \pm}$. It is important to notice that for a CP-even or a CP-odd Higgs boson, the position of the maximum of the $\varphi_{\mathrm{CP}}^{*}$ distribution, whose true value is at $\varphi_{\mathrm{CP}}^{*}=0$, respectively at $\varphi_{\mathrm{CP}}^{*}=\pi$, is neither affected by the smearing procedure nor by a cut on the impact parameters.

Next we apply the same smearing procedure and cuts also to a Higgs boson $h$ being a CP mixture with $\phi_{\tau}=-\pi / 4$. The resulting $\varphi_{\mathrm{CP}}^{*}$ distributions are shown in the left plot of Fig. 8. The asymmetry (18), whose NLO QCD value for this decay mode is $A_{\mathrm{NLO}}^{\pi \pi}=49.5 \%\left(A_{\mathrm{LO}}^{\pi \pi}=50.2 \%\right)$ is reduced to $29 \%\left(A_{\mathrm{LO}}^{\pi \pi}=29.3 \%\right)$ by the measurement uncertainties as specified above. By applying a minimum cut on both impact parameters $n_{ \pm}$the asymmetry can be enhanced to $36.7,42,46.6 \%$ for $n_{ \pm} \geq 20,30,40 \mu \mathrm{m}$. More importantly, however, the position of the maximum of the $\varphi_{\mathrm{CP}}^{*}$ distribution turns out to depend on the smearing parameters and on the cut on $n_{ \pm}$. In the case of no smearing the position of the maximum is at $\varphi_{C P, \max }^{*}=\pi / 2=1.57$. With smearing and no cut on $n_{ \pm}$the location of the maximum moves to $\varphi_{C P, \max }^{*}=1.24$. For $n_{ \pm} \geq 20,30,40 \mu \mathrm{m}$ the maximum is at $\varphi_{C P, \max }^{*}=1.3,1.36,1.4$. The reason for this shift of the maximum is the smearing of the primary vertex. For larger values of $\sigma_{z}^{P V}$ or $\sigma_{t r}^{P V}$ the reconstructed PV moves further away from the two tracks of $\pi^{ \pm}$. Therefore, the angle between the two impact parameters becomes smaller. This leads to an enhancement of the $\varphi_{\mathrm{CP}}^{*}$ distribution near $\varphi_{\mathrm{CP}}^{*} \sim 0$ and $\varphi_{\mathrm{CP}}^{*} \sim 2 \pi$. In the case of smearing, $p_{T}^{\pi}$ cuts affect also the position of the maximum of the $\varphi_{\mathrm{CP}}^{*}$ distribution. Because the Higgs mixing angle $\phi_{\tau}$ is determined from the difference between the position $\varphi_{C P, \max }^{*}$ of the maximum of the measured distribution and $\varphi_{\mathrm{CP}}^{*}=\pi$ (cf. Fig. 1) it is crucial to 


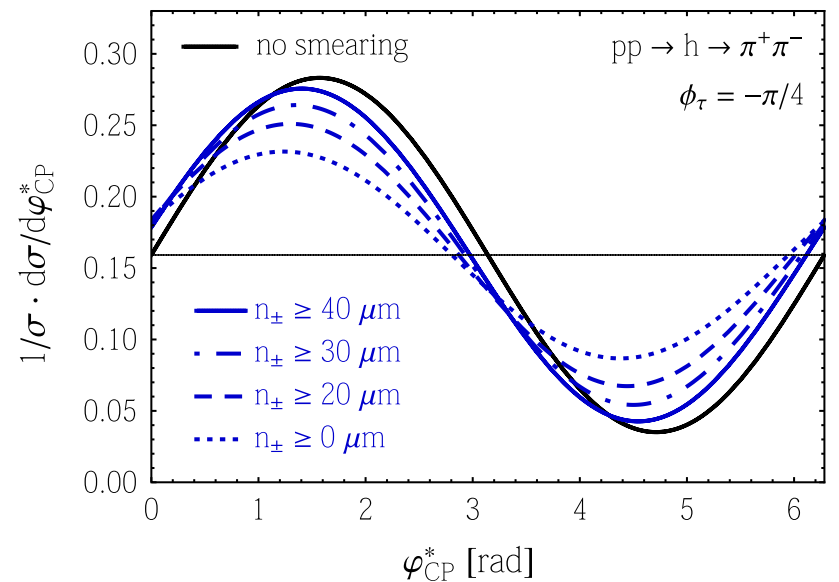

Fig. $8 \mathrm{LHC}(\sqrt{S}=14 \mathrm{TeV}), p p \rightarrow h+X$, for a CP mixture $h$ with mixing angle $\phi_{\tau}=-\pi / 4$. Left: $h \rightarrow \tau^{-} \tau^{+} \rightarrow \pi^{+} \pi^{-}$with $p_{T}^{\pi} \geq 20 \mathrm{GeV},\left|\eta_{\pi}\right| \leq 2.5$. The normalized $\varphi_{\mathrm{CP}}^{*}$ distribution, taking measurement uncertainties into account, for different minimum cuts on

understand the measurement uncertainties. As will be shown in the next section, the measurement of the $\varphi_{\mathrm{CP}}^{*}$ distribution for Drell-Yan $\tau$-pair production can be used to get a handle on these uncertainties.

This shift of the maximum of the $\varphi_{\mathrm{CP}}^{*}$ distribution, which occurs for a CP mixture, can become even larger for decay modes such as $\rho \rho$ or $\rho a_{1}$ if additional cuts on $\tilde{E}_{ \pm}$are applied. As an example, we consider the $h \rightarrow \tau^{-} \tau^{+} \rightarrow \rho^{-} \rho^{+} \rightarrow$ $\pi^{-} \pi^{+}$decay channel for a CP mixture $h$ with $\phi_{\tau}=-\pi / 4$. We apply the cuts $\tilde{E}_{ \pm} \geq 30 \mathrm{GeV}$ in order to obtain a large asymmetry (18), cf. Sect. 6.2.2. The solid black curve in Fig. 8, right, shows the associated normalized $\varphi_{\mathrm{CP}}^{*}$ distribution without smearing. Taking measurement uncertainties into account one obtains the distribution given by the blue dashed line. Its maximum is shifted from $\varphi_{\mathrm{CP}, \max }^{*}=\pi / 2$ to $\varphi_{\mathrm{CP} \text {,max }}^{*} \simeq 1$. Notice that the smeared distribution is raised at $\varphi_{\mathrm{CP}}^{*} \sim 0$ and $\varphi_{\mathrm{CP}}^{*} \sim 2 \pi$ and lowered at $\varphi_{\mathrm{CP}}^{*} \sim \pi$ as compared to the unsmeared distribution. This is due to the smearing of the PV. The PV uncertainty causes the same effect on the $\varphi_{\mathrm{CP}}^{*}$ distributions of the other $\rho \rho \rightarrow \pi \pi$ event categories, e.g. for events with $\tilde{E}_{-} \geq 30 \mathrm{GeV}$ and $\tilde{E}_{+} \leq 30 \mathrm{GeV}$, and of other $\tau \tau \rightarrow a a^{\prime}$ decay modes. At this point we notice that an increase of the uncertainties of the other parameters discussed above makes the $\varphi_{\mathrm{CP}}^{*}$ distributions flatter.

\subsection{The $\varphi_{\mathrm{CP}}^{*}$ distribution for Drell-Yan production of $\tau$ pairs}

In this section we investigate the impact of measurement uncertainties on the $\varphi_{\mathrm{CP}}^{*}$ distribution for the Drell-Yan production of $\tau \tau$. If not stated otherwise we use, as above, for the smeared distributions the parameters $\sigma_{z}^{P V}=20 \mu \mathrm{m}, \sigma_{t r}^{P V}=$ $10 \mu \mathrm{m}, \sigma_{t r}^{\pi}=10 \mu \mathrm{m}, \sigma_{\theta}^{\pi}=1 \mathrm{mrad}$ and $\Delta E^{\pi} / E^{\pi}=5 \%$.

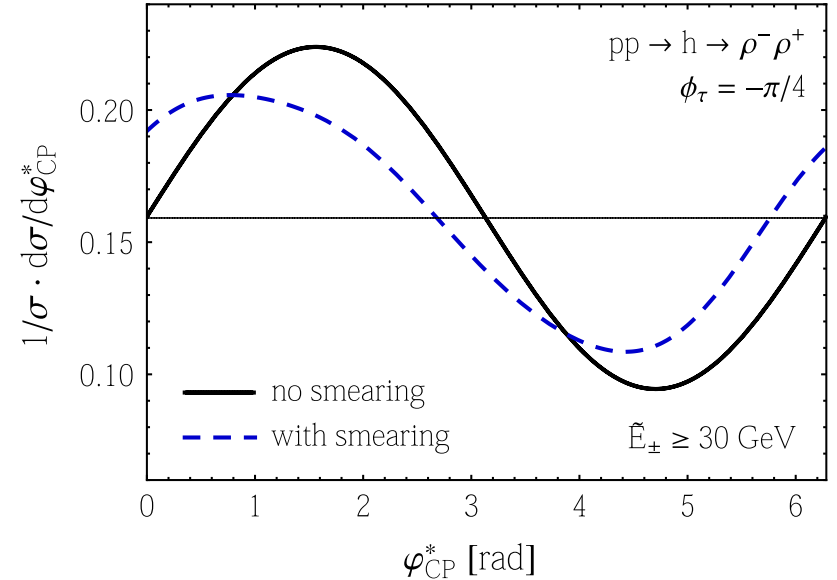

the length of the impact parameters $n_{ \pm}$. Right: $p p \rightarrow h \rightarrow \tau^{-} \tau^{+} \rightarrow$ $\rho^{-} \rho^{+} \rightarrow \pi^{-} \pi^{+}$. The normalized $\varphi_{\mathrm{CP}}^{*}$ distribution for events with $\tilde{E}_{ \pm} \geq 30 \mathrm{GeV}$ in the $\tau \tau \mathrm{ZMF}$, without and with smearing. The horizontal lines in both plots are drawn to guide the eye

Furthermore, we apply the cut $n_{ \pm} \geq 20 \mu \mathrm{m}$ in the computation of the distributions of this section. For definiteness, we consider the normalized $\varphi_{\mathrm{CP}}^{*}$ distribution for the $p p \rightarrow Z^{*} / \gamma^{*} \rightarrow \tau^{-} \tau^{+} \rightarrow \pi^{+} \pi^{-}+2 v$ production mode. Without smearing the distribution is given by the dotted black flat line in Fig. 9, left, while the effect of the PV uncertainty, simulated with $\sigma_{z}^{P V}=20 \mu \mathrm{m}$ and with $\sigma_{z}^{P V}=40 \mu \mathrm{m}$, results in the distribution shown by the dashed and solid blue line, respectively. The shape of these curves can be understood as follows. If the measurement uncertainty of the PV becomes larger, the distance of the reconstructed PV to each of the tracks of the charged pions $\pi^{ \pm}$increases. This results in a smaller angle between the two reconstructed impact parameter vectors. This, in turn, enhances the region of $\varphi_{\mathrm{CP}}^{*} \sim 0$ and $\varphi_{\mathrm{CP}}^{*} \sim 2 \pi$ in the $\varphi_{\mathrm{CP}}^{*}$ distribution. On the other hand we found that larger values of $\sigma_{t r}^{\pi}, \sigma_{\theta}^{\pi}$ and $\Delta E^{\pi}$ decrease the curvature of the smeared distribution.

An important result of our simulation of the smeared normalized $\varphi_{\mathrm{CP}}^{*}$ distributions for $h \rightarrow \tau \tau$ and $Z^{*} / \gamma^{*} \rightarrow \tau \tau$ is that they are both enhanced (for $h$ of arbitrary $\mathrm{CP}$ nature) at $\varphi_{\mathrm{CP}}^{*} \sim 0$ and $\varphi_{\mathrm{CP}}^{*} \sim 2 \pi$ as compared to the respective unsmeared distribution - an effect which is due to the finite experimental resolution of the primary vertex. Based on this result we suggest the following procedure to obtain a $\varphi_{\mathrm{CP}}^{*}$ distribution for the signal reactions $h \rightarrow \tau \tau \rightarrow a a^{\prime}$, with which these distortions can be eliminated to a large extent. We assume that a clean data sample of Drell-Yan $\tau$ pair events can be recorded at the LHC. One measures the normalized $\varphi_{\mathrm{CP}}^{*}$ distribution for this sample and subtracts it from the distribution measured with the $\tau \tau$ events in the signal region, $M_{\tau \tau} \sim 125 \mathrm{GeV}$.

We exemplify this proposal for $h, Z^{*} / \gamma^{*} \rightarrow \tau^{-} \tau^{+} \rightarrow$ $\rho^{-} \rho^{+} \rightarrow \pi^{-} \pi^{+}$, taking $h$ to be a CP mixture with $\phi_{\tau}=$ $-\pi / 4$. Figure 9 , right, shows the unsmeared and smeared 


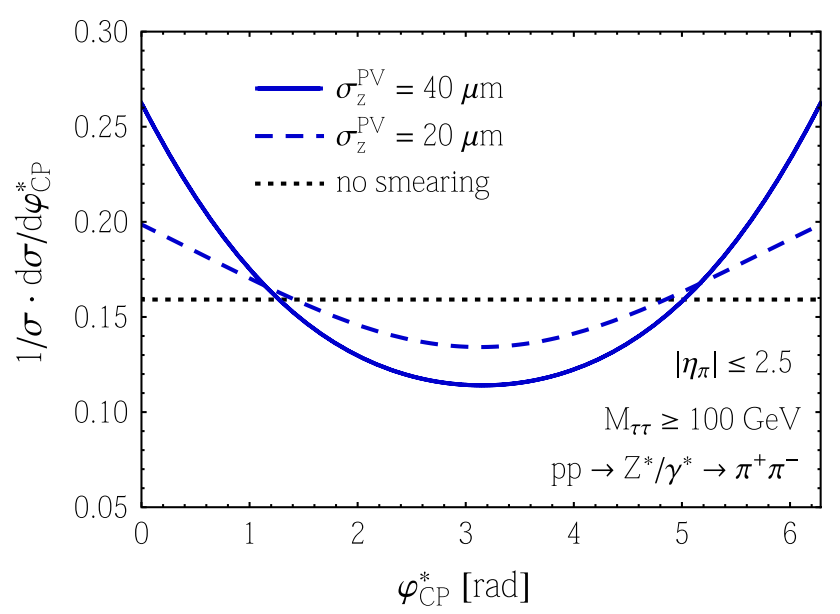

Fig. 9 Left: $p p \rightarrow Z^{*} / \gamma^{*} \rightarrow \tau^{-} \tau^{+} \rightarrow \pi^{+} \pi^{-}$with $p_{T}^{\pi} \geq 20 \mathrm{GeV}$ and $n_{ \pm} \geq 20 \mu \mathrm{m}$. Normalized smeared $\varphi_{\mathrm{CP}}^{*}$ distribution for two different values of $\sigma_{z}^{P V}$. The dotted black line is the prediction with-

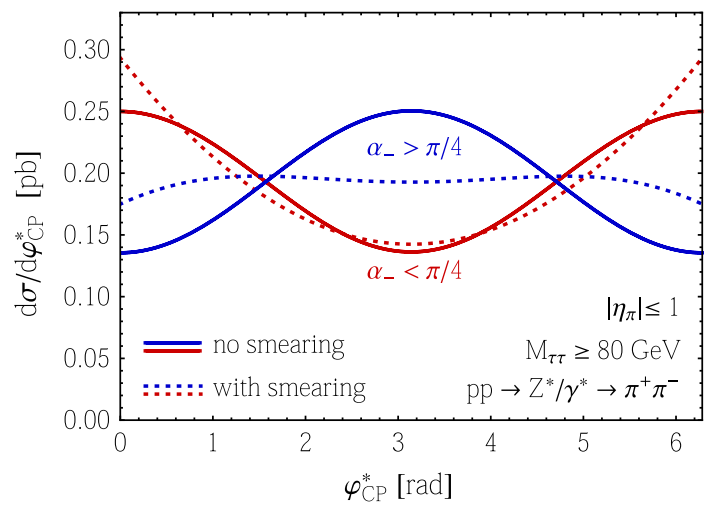

Fig. $10 p p \rightarrow Z^{*} / \gamma^{*} \rightarrow \tau^{-} \tau^{+} \rightarrow \pi^{+} \pi^{-}+2 \nu$. (Un)smeared $\varphi_{\mathrm{CP}}^{*}$ distributions for events with $\pi^{-}$being nearly coplanar $\left(\alpha_{-}<\pi / 4\right)$ and events with $\pi^{-}$being nearly perpendicular $\left(\alpha_{-}>\pi / 4\right)$

$\varphi_{\mathrm{CP}}^{*}$ distributions for $h$ decay and the smeared distribution for $Z^{*} / \gamma^{*}$ decay. Subtracting the latter distribution from the smeared distribution for $h$ decay and adding the flat line $\sigma^{-1} d \sigma / d \varphi_{\mathrm{CP}}^{*}=1 /(2 \pi)$, one obtains the solid blue curve. The maximum of this corrected signal distribution is at $\varphi_{\mathrm{CP}}^{*, \max }=\pi / 2$ where it should be. This illustrates that with this procedure, one gets rid of the distortions caused by the PV measurement uncertainties to a large extent.

Finally, we compute for $p p \rightarrow Z^{*} / \gamma^{*} \rightarrow \tau^{-} \tau^{+} \rightarrow$ $\pi^{+} \pi^{-}+2 v$ the smeared $\varphi_{\mathrm{CP}}^{*}$ distribution for events with $\pi^{-}$being nearly coplanar $\left(\alpha_{-}<\pi / 4\right)$ and events with $\pi^{-}$ being nearly perpendicular $\left(\alpha_{-}>\pi / 4\right)$, cf. Sect. 5 . The distribution for $\alpha_{-}>\pi / 4$ gets significantly distorted by the smearing, as shown in Fig. 10. As already discussed in Sect. 5 we propose to measure these two distributions as a means to calibrate the signal distribution.

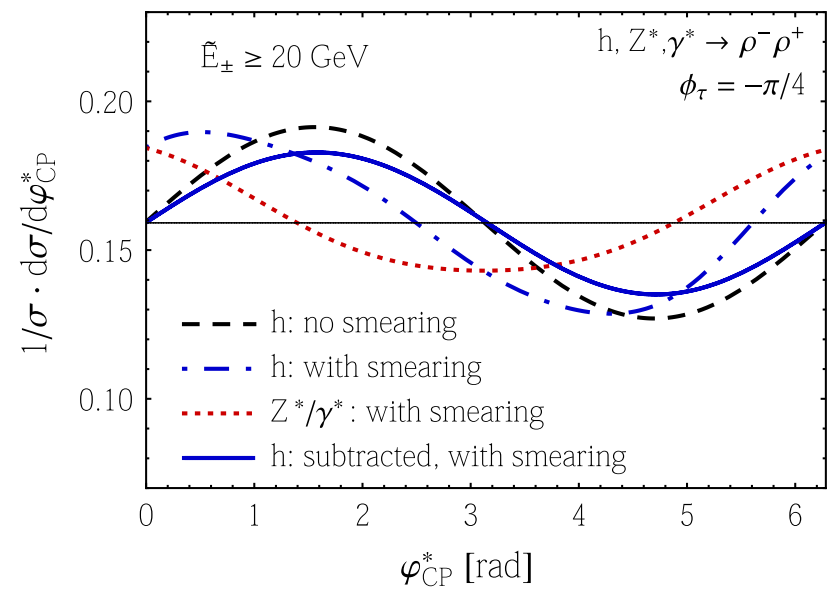

out measurement uncertainties. Right: decays $h, Z^{*} / \gamma^{*} \rightarrow \tau^{-} \tau^{+} \rightarrow$ $\rho^{-} \rho^{+} \rightarrow \pi^{-} \pi^{+}$, taking $h$ to be a CP mixture with $\phi_{\tau}=-\pi / 4$. Normalized (un)smeared $\varphi_{\mathrm{CP}}^{*}$ distributions for events with $\tilde{E}_{ \pm} \geq 20 \mathrm{GeV}$

\subsection{Estimate of $\Delta \phi_{\tau}$}

In this section we estimate the statistical uncertainty $\Delta \phi_{\tau}$ with which the mixing angle $\phi_{\tau}$ can be determined from the smeared $\varphi_{\mathrm{CP}}^{*}$ distributions of the $h \rightarrow \tau \tau$ decay modes, taking into account the $\tau$ decay channels (3)-(7). As discussed above, the asymmetry $A^{a a^{\prime}}$ in Eq. (18) is a measure of the statistical uncertainty $\Delta \phi_{\tau}$ for each decay channel $a a^{\prime}$, for a given number of events. ${ }^{4}$

For each $\tau$-decay mode the asymmetry (18) is calculated by generating the smeared $\varphi_{\mathrm{CP}}^{*}$ distribution of the Higgs-boson signal and of the $Z^{*} / \gamma^{*}$ background with our Monte Carlo program, using the smearing parameters $\sigma_{z}^{P V}=20 \mu \mathrm{m}, \sigma_{t r}^{P V}=10 \mu \mathrm{m}, \sigma_{t r}^{\pi}=10 \mu \mathrm{m}, \sigma_{\theta}^{\pi}=1 \mathrm{mrad}$, $\Delta E^{\pi} / E^{\pi}=5 \%$, the cut $n_{ \pm} \geq 20 \mu m$ on the length of the impact parameters and the cut $M_{\tau \tau} \geq 100 \mathrm{GeV}$. Furthermore, for the leptonic decay modes we apply the chargedlepton cuts $p_{T, l} \geq 20 \mathrm{GeV}$ and $\left|\eta_{l}\right| \leq 2.5$. For the hadronic decay modes the cuts $p_{T, \tau} \geq 20 \mathrm{GeV}$ and $\left|\eta_{\tau}\right| \leq 2.5$ are used, which approximate roughly corresponding cuts on the hadronic $\tau$ jets used in experiments. As discussed in the last section, we correct the normalized Higgs-boson $\varphi_{\mathrm{CP}}^{*}$ distribution, for each decay channel, by subtracting the normalized $\varphi_{\mathrm{CP}}^{*, Z \gamma}$ distribution and adding the flat distribution $1 /(2 \pi)$. The resulting distribution is then reweighted in order for it to be properly normalized. From this distribution we calculate the signal asymmetry $A_{S}$. It is given for the finalstate event categories 'hadron-hadron' (had-had), 'lepton-

\footnotetext{
$\overline{4}$ The analysis of the various decays $h \rightarrow \tau \tau \rightarrow$ charged prongs made here is analogous to our investigation in [67], where Higgs-boson production in $e^{+} e^{-}$collisions by $e^{+} e^{-} \rightarrow Z h$ was considered. In the present analysis, the cuts on the energies of the pions and charged leptons are made in the Higgs-boson rest frame.
} 
Table 1 Asymmetries and signal reduction for the hadron-hadron, lepton-hadron, and lepton-lepton decay modes. The estimate of "events/fb" includes background events

\begin{tabular}{llllll}
\hline $\begin{array}{l}\tau \tau \text { Decay } \\
\text { channel }\end{array}$ & $A_{S}[\%]$ & $\frac{S}{S+B}$ & $A_{S+B}[\%]$ & $\sigma_{n_{ \pm} \geq 20 \mu m} / \sigma$ & Events $/ f b$ \\
\hline had-had & 13.2 & 0.5 & 6.6 & 0.58 & 1.16 \\
lep-had & 9.0 & 0.5 & 4.5 & 0.63 & 1.26 \\
lep-lep & 7.0 & $1 / 3$ & 2.3 & 0.61 & 1.22 \\
Combined & & & 4.85 & 0.61 & 3.66 \\
\hline
\end{tabular}

hadron' (lep-had), and 'lepton-lepton' (lep-lep) in column 2 of Table 1 . The asymmetry for signal plus background is then obtained by $A_{S+B}=A_{S} \times S /(S+B)$.

In order to estimate the number of events including the background that may be available for $\varphi_{\mathrm{CP}}^{*}$ measurements at the LHC (14 TeV), we assume for the hadron-hadron and lepton-hadron decay channels the ratio $S / B=1$ and $^{5} S+$ $B=2$ events $/ f b[8]$. For the lepton-lepton decay modes we assume $S / B=1 / 2$ and $S+B=2$ events $/ f b$. The resulting ratios $S /(S+B)$ and asymmetries $A_{S+B}$ are given in column 3 and 4 of Table 1 . Next we calculate the factor $R_{n}=$ $\sigma_{n_{ \pm} \geq 20 \mu m} / \sigma$ by which the respective signal cross section is reduced by a cut on the impact parameters. These factors are given in column 5 of Table 1 . The number of events/fb is then given by $(S+B) \times R_{n}$, cf. column 6 of Table 1 .

With these values of the asymmetry $A_{S+B}$ and number of events/fb for the different event categories introduced above, we estimate the statistical uncertainty $\Delta \phi_{\tau}$ in the following way [38]. We choose some value of the Higgs mixing angle, for example $\phi_{\tau}=-\pi / 4$, and generate the corresponding differential $\varphi_{\mathrm{CP}}^{*}$ distribution using 20 bins between 0 and $2 \pi$. We then fit this distribution with the function $u \cos \left(\varphi_{\mathrm{CP}}^{*}-\right.$ $\left.2 \phi_{\tau}\right)+v$. This is repeated a sufficiently large number of times $(\sim 1,000$ times). In this way we obtain a distribution of the values of $\phi_{\tau}$ extracted from these fits. This $\phi_{\tau}$ distribution is fitted with a Gaussian, and we take its width as our estimate of the statistical uncertainty $\Delta \phi_{\tau}$. The result of this procedure is shown in Fig. 11. The gray contour lines display $\Delta \phi_{\tau}$ as a function of $A_{S+B}$ and the number of events. The horizontal lines are the asymmetries $A_{S+B}$ for the three event categories and their combination. Assuming that at the LHC (14 TeV) an integrated luminosity of $150 \mathrm{fb}^{-1}, 500 \mathrm{fb}^{-1}$, and $3 a b^{-1}$ will be achieved - the two last numbers are goals for the highluminosity LHC upgrade [101] — the resulting event numbers are sketched in as black, red, and yellow dots, respectively. The yellow dot on the line for the combined asymmetry, which corresponds to 11000 events, is not shown. For these

\footnotetext{
5 We have extracted these numbers from the results of the ATLAS experiment for the hadron-hadron and lepton-hadron channels in $h \rightarrow \tau \tau$ at the LHC (8 TeV) [8]. The actual number of events at the LHC (14 TeV) will be higher-however, the $S / B$ ratio will be worse.
}

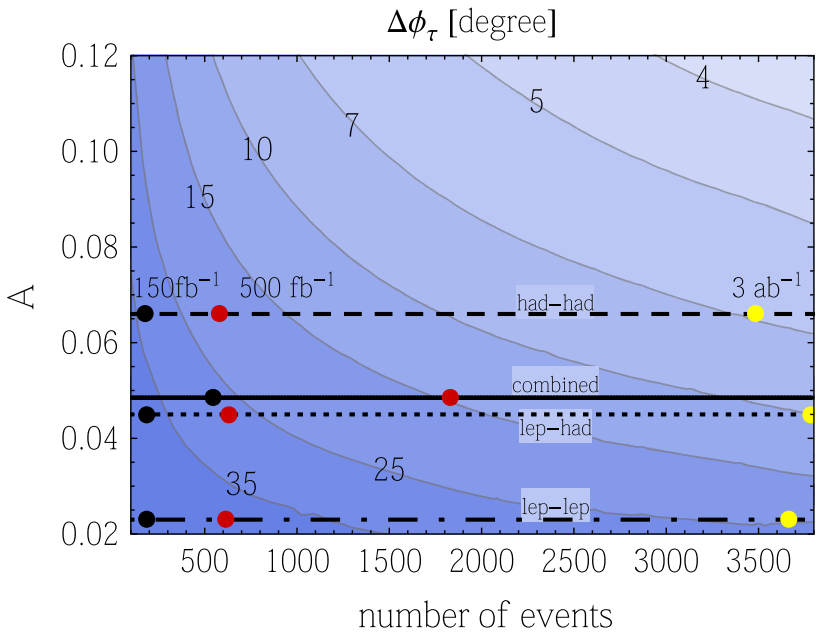

Fig. $11 \mathrm{LHC}(\sqrt{S}=14 \mathrm{TeV})$. Estimated statistical uncertainty $\Delta \phi_{\tau}$ of the Higgs mixing angle $\phi_{\tau}$ as a function of the number of events (signal + background). The horizontal lines display the asymmetries $A_{S+B}$ of the three event categories given in Table 1. The yellow dot on the solid black line is not shown, it corresponds to 11000 events

luminosities our estimate of the statistical uncertainty $\Delta \phi_{\tau}$ which can be achieved by using the combination of the three event categories is $27^{\circ}, 14.3^{\circ}$, and $5.1^{\circ}$, respectively.

\section{Summary}

We have investigated how precisely the $\mathrm{CP}$ nature of the $125 \mathrm{GeV}$ Higgs-boson resonance $h$ can be determined at the LHC (14 TeV) in its decay to $\tau$ leptons. As to the subsequent $\tau$ decays, we have taken into account all the major decay modes (3)-(7). Our method for determining the Higgs mixing angle $\phi_{\tau}$, which parameterizes the ratio of the reduced pseudoscalar and scalar Higgs- $\tau$ Yukawa couplings, is based on the distribution of the angle $\varphi_{\mathrm{CP}}^{*}$ defined in (17). This distribution can be measured for all charged-prong $\tau$ decays without having to reconstruct the $\tau^{\mp}$ rest frames. For definiteness, we have considered inclusive Higgs-boson production by gluon fusion. The irreducible background from Drell-Yan production of $\tau$ pairs was analyzed in detail, in particular its contribution to the $\varphi_{\mathrm{CP}}^{*}$ distribution. We have studied by Monte Carlo simulation how measurement uncertainties affect the signal and background contributions to this distribution. Based on this study we devised a procedure for obtaining a corrected distribution of this angle. This procedure eliminates to a large extent the distortions due to the measurement uncertainty of the Higgs production vertex. Moreover, we made a proposal how to use the $\varphi_{\mathrm{CP}}^{*}$ distribution of Drell-Yan $\tau$-pair events for calibrating the experimental uncertainties. Taking the background from $Z^{*} / \gamma^{*} \rightarrow \tau \tau$ and measurement uncertainties by Monte Carlo simulation into account, we found that at the LHC (14 TeV), respectively at the LHC-upgrade, with an 
integrated luminosity of $150 \mathrm{fb}^{-1}, 500 \mathrm{fb}^{-1}$, and $3 a b^{-1}$, the Higgs mixing angle $\phi_{\tau}$ can be determined with a precision $\Delta \phi_{\tau}$ of $27^{\circ}, 14.3^{\circ}$, and $5.1^{\circ}$.

Acknowledgments We wish to thank the members of the $M_{\tau \tau}$ working group of the Helmholtz Alliance "Physics at the Terascale" for discussions. The work of W.B. is supported by B.M.B.F., contract 05H12PAE, and S.K. is supported by Deutsche Forschungsgemeinschaft through Graduiertenkolleg GRK 1675.

Open Access This article is distributed under the terms of the Creative Commons Attribution License which permits any use, distribution, and reproduction in any medium, provided the original author(s) and the source are credited.

Funded by $\mathrm{SCOAP}^{3}$ / License Version CC BY 4.0.

\section{References}

1. G. Aad et al., ATLAS Collaboration, Phys. Lett. B 716, 1 (2012). arXiv:1207.7214 [hep-ex]

2. S. Chatrchyan et al., CMS Collaboration. Phys. Lett. B 716, 30 (2012). arXiv:1207.7235 [hep-ex]

3. S. Chatrchyan et al. [CMS Collaboration], Phys. Rev. Lett. 110, 081803 (2013). arXiv:1212.6639 [hep-ex]

4. G. Aad et al. [ATLAS Collaboration], Phys. Lett. B 726, 120 (2013). arXiv:1307.1432 [hep-ex]

5. S. Chatrchyan et al. [CMS Collaboration], JHEP 06, 081 (2013). arXiv:1303.4571 [hep-ex]

6. G. Aad et al. [ATLAS Collaboration], Phys. Lett. B 726, 88 (2013). arXiv: 1307.1427 [hep-ex]

7. S. Chatrchyan et al. [CMS Collaboration]. arXiv:1312.5353 [hepex]

8. ATLAS Collaboration, conference note ATLAS-CONF-2013-108

9. S. Chatrchyan et al. [CMS Collaboration]. arXiv:1401.6527 [hepex]

10. S. Chatrchyan et al. [CMS Collaboration]. arXiv:1401.5041 [hepex]

11. ATLAS Collaboration, conference note ATLAS-CONF-2014-009

12. J.R. Dell'Aquila, C.A. Nelson, Phys. Rev. D 33, 93 (1986)

13. J.R. Dell'Aquila, C.A. Nelson, Nucl. Phys. B 320, 61 (1989)

14. W. Bernreuther, A. Brandenburg, Phys. Lett. B 314, 104 (1993)

15. W. Bernreuther, A. Brandenburg, Phys. Rev. D 49, 4481 (1994). hep-ph/9312210

16. A. Soni, R.M. Xu, Phys. Rev. D 48, 5259 (1993). hep-ph/9301225

17. D. Chang, W.-Y. Keung, I. Phillips, Phys. Rev. D 48, 3225 (1993). hep-ph/9303226

18. V.D. Barger, K. Cheung, A. Djouadi, B.A. Kniehl, P.M. Zerwas, Phys. Rev. D 49, 79 (1994). hep-ph/9306270

19. M. Krämer, J.H. Kühn, M.L. Stong, P.M. Zerwas, Z. Phys, C 64, 21 (1994). hep-ph/9404280

20. K. Hagiwara, M.L. Stong, Z. Phys, C 62, 99 (1994). hep-ph/9309248

21. T. Arens, U.D.J. Gieseler, L.M. Sehgal, Phys. Lett. B 339, 127 (1994). hep-ph/9408316

22. A. Skjold, P. Osland, Phys. Lett. B 329, 305 (1994). hep-ph/9402358

23. T. Arens, L.M. Sehgal, Z. Phys, C 66, 89 (1995). hep-ph/9409396

24. A. Skjold, P. Osland, Nucl. Phys. B 453, 3 (1995). hep-ph/9502283

25. S. Bar-Shalom, D. Atwood, G. Eilam, R.R. Mendel, A. Soni, Phys. Rev. D 53, 1162 (1996). hep-ph/9508314

26. B. Grzadkowski, J.F. Gunion, Phys. Lett. B 350, 218 (1995). hep-ph/9501339
27. J.F. Gunion, B. Grzadkowski, X.-G. He, Phys. Rev. Lett. 77, 5172 (1996). hep-ph/9605326

28. W. Bernreuther, A. Brandenburg, M. Flesch, Phys. Rev. D 56, 90 (1997). hep-ph/9701347

29. S. Bar-Shalom, D. Atwood, A. Soni, Phys. Lett. B 419, 340 (1998). hep-ph/9707284

30. B. Grzadkowski, J.F. Gunion, J. Kalinowski, Phys. Rev. D 60, 075011 (1999). hep-ph/9902308

31. K. Hagiwara, S. Ishihara, J. Kamoshita, B.A. Kniehl, Eur. Phys. J. C 14, 457 (2000). hep-ph/0002043

32. T. Han, J. Jiang, Phys. Rev. D 63, 096007 (2001). hep-ph/0011271

33. T. Plehn, D.L. Rainwater, D. Zeppenfeld, Phys. Rev. Lett. 88, 051801 (2002). hep-ph/0105325

34. S.Y. Choi, D.J. Miller, M.M. Mühlleitner, P.M. Zerwas, Phys. Lett. B 553, 61 (2003). hep-ph/0210077

35. G.R. Bower, T. Pierzchala, Z. Was, M. Worek, Phys. Lett. B 543, 227 (2002). hep-ph/0204292

36. K. Desch, Z. Was, M. Worek, Eur. Phys. J. C 29, 491 (2003). hep-ph/0302046

37. E. Asakawa, K. Hagiwara, Eur. Phys. J. C 31, 351 (2003). hep-ph/0305323

38. K. Desch, A. Imhof, Z. Was, M. Worek, Phys. Lett. B 579, 157 (2004). hep-ph/0307331

39. C.P. Buszello, I. Fleck, P. Marquard, J.J. van der Bij, Eur. Phys. J. C 32, 209 (2004). hep-ph/0212396

40. R.M. Godbole, S. Kraml, M. Krawczyk, D.J. Miller, P. Niezurawski, A.F. Zarnecki. hep-ph/0404024

41. A. Rougé, Phys. Lett. B 619, 43 (2005). hep-ex/0505014

42. S.S. Biswal, R.M. Godbole, R.K. Singh, D. Choudhury, Phys. Rev. D 73, 035001 (2006) [Erratum-ibid. D 74039904 (2006)]. hep-ph/0509070

43. J.R. Ellis, J.S. Lee, A. Pilaftsis, Phys. Rev. D 72, 095006 (2005). hep-ph/0507046

44. E. Accomando et al. hep-ph/0608079

45. R.M. Godbole, D.J. Miller, M.M. Mühlleitner, JHEP 0712, 031 (2007). arXiv:0708.0458 [hep-ph]

46. P.S. Bhupal Dev, A. Djouadi, R.M. Godbole, M. Mühlleitner, S.D. Rindani, Phys. Rev. Lett. 100051801 (2008). arXiv:0707.2878 [hep-ph]

47. G. Klamke, D. Zeppenfeld, JHEP 0704052 (2007). hep-ph/0703202 [HEP-PH]

48. S. Berge, W. Bernreuther, J. Ziethe, Phys. Rev. Lett. 100, 171605 (2008). arXiv:0801.2297 [hep-ph]

49. S. Dutta, K. Hagiwara, Y. Matsumoto, Phys. Rev. D 78, 115016 (2008). arXiv:0808.0477 [hep-ph]

50. S.S. Biswal, D. Choudhury, R.M. Godbole, Mamta, Phys. Rev. D 79035012 (2009). arXiv:0809.0202 [hep-ph]

51. S. Berge, W. Bernreuther, Phys. Lett. B 671, 470 (2009). arXiv:0812.1910 [hep-ph]

52. M. Reinhard, $C P$ violation in the Higgs sector with a nextgeneration detector at the ILC, PhD thesis, LLR -École Polytechnique, November 2009

53. H. Videau, Measuring the CP state of the Higgs through its decay H to tau tau, IWLC2010 (International Workshop on Linear Colliders 2010) at CERN, https://espace.cern.ch/LC2010/default. aspx

54. A. De Rujula, J. Lykken, M. Pierini, C. Rogan, M. Spiropulu, Phys. Rev. D 82, 013003 (2010). arXiv: 1001.5300 [hep-ph]

55. N.D. Christensen, T. Han, Y. Li, Phys. Lett. B 693, 28 (2010). arXiv: 1005.5393 [hep-ph]

56. S. Berge, W. Bernreuther, B. Niepelt, H. Spiesberger, Phys. Rev. D 84, 116003 (2011). arXiv:1108.0670 [hep-ph]

57. R.M. Godbole, C. Hangst, M. Mühlleitner, S.D. Rindani, P. Sharma, Eur. Phys. J. C 71, 1681 (2011). arXiv:1103.5404 [hep$\mathrm{ph}]$ 
58. S.S. Biswal, R.M. Godbole, B. Mellado, S. Raychaudhuri, Phys. Rev. Lett. 109, 261801 (2012). arXiv:1203.6285 [hep-ph]

59. J. Ellis, D.S. Hwang, V. Sanz, T. You, JHEP 1211, 134 (2012). arXiv:1208.6002 [hep-ph]

60. S. Banerjee, J. Kalinowski, W. Kotlarski, T. Przedzinski, Z. Was, Eur. Phys. J. C 73, 2313 (2013). arXiv:1212.2873 [hep-ph]

61. K. Hagiwara, T. Li, K. Mawatari, J. Nakamura, Eur. Phys. J. C 73, 2489 (2013). arXiv:1212.6247 [hep-ph]

62. A. Djouadi, R.M. Godbole, B. Mellado, K. Mohan, Phys. Lett. B 723, 307 (2013). arXiv:1301.4965 [hep-ph]

63. C. Englert, D. Goncalves, G. Nail, M. Spannowsky, Phys. Rev. D 88, 013016 (2013). arXiv:1304.0033 [hep-ph]

64. B. Ananthanarayan, S.K. Garg, J. Lahiri, P. Poulose, Phys. Rev. D 87, 114002 (2013). arXiv:1304.4414 [hep-ph]

65. J. Frank, M. Rauch, D. Zeppenfeld. arXiv:1305.1883 [hep-ph]

66. R. Harnik, A. Martin, T. Okui, R. Primulando, F. Yu, Phys. Rev. D 88(7), 076009 (2013). arXiv:1308.1094 [hep-ph]

67. S. Berge, W. Bernreuther, H. Spiesberger, Phys. Lett. B 727, 488 (2013). arXiv:1308.2674 [hep-ph]

68. F. Bishara, Y. Grossman, R. Harnik, D.J. Robinson, J. Shu, J. Zupan, JHEP 1404, 084 (2014). arXiv:1312.2955 [hep-ph]

69. J. Ellis, D.S. Hwang, K. Sakurai, M. Takeuchi, JHEP 1404, 004 (2014). arXiv:1312.5736 [hep-ph]

70. A. Kaczmarska, J. Piatlicki, T. Przedzinski, E. Richter-Was, Z. Was, arXiv: 1402.2068 [hep-ph]

71. T. Przedzinski, E. Richter-Was, Z. Was. arXiv:1406.1647 [hep$\mathrm{ph}]$

72. Y. Chen, A. Falkowski, I. Low, R. Vega-Morales. arXiv:1405.6723 [hep-ph]

73. J. Kopp, M. Nardecchia. arXiv:1406.5303 [hep-ph]

74. M.J. Dolan, P. Harris, M. Jankowiak, M. Spannowsky. arXiv:1406.3322 [hep-ph]

75. F. Demartin, F. Maltoni, K. Mawatari, B. Page, M. Zaro. arXiv: 1407.5089 [hep-ph]

76. A. Djouadi, G. Moreau, Eur. Phys. J. C 73, 2512 (2013). arXiv:1303.6591 [hep-ph]

77. P. Bechtle, S. Heinemeyer, O. Stal, T. Stefaniak, G. Weiglein, Eur. Phys. J. C 74, 2711 (2014). arXiv:1305.1933 [hep-ph]

78. J. Brod, U. Haisch, J. Zupan, JHEP 1311, 180 (2013). arXiv:1310.1385 [hep-ph]

79. P. Bechtle, S. Heinemeyer, O. Stal, T. Stefaniak, G. Weiglein. arXiv:1403.1582 [hep-ph]
80. S. Inoue, M.J. Ramsey-Musolf, Y.Zhang, Phys. Rev. D 89, 115023 (2014). arXiv:1403.4257 [hep-ph]

81. J.M. Campbell, R.K. Ellis, Nucl. Phys. Proc. Suppl. 205-206, 10 (2010). arXiv:1007.3492 [hep-ph]

82. J.M. Campbell, R.K. Ellis, C. Williams. http://mcfm.fnal.gov

83. A. Rougé, Z. Phys C 48, 75 (1990)

84. M. Davier, L. Duflot, F. Le Diberder, A. Rougé, Phys. Lett. B 306, 411 (1993)

85. J.H. Kühn, Phys. Rev. D 52, 3128 (1995)

86. A. Stahl, Springer Tracts Mod. Phys. 160, 1 (2000)

87. W. Bernreuther, T. Schröder, T.N. Pham, Phys. Lett. B 279, 389 (1992)

88. CMS Collaboration, CMS-PAS-HIG-14-002

89. S. Dittmaier et al. [LHC Higgs Cross Section Working Group Collaboration]. arXiv:1101.0593 [hep-ph]

90. W. Bernreuther, O. Nachtmann, P. Overmann, Phys. Rev. D 48, 78 (1993)

91. S. Catani, M.H. Seymour, Nucl. Phys. B 485, 291 (1997) rratumibid. B 510, 503 (1998)]. hep-ph/9605323

92. J.M. Campbell, R.K. Ellis, G. Zanderighi, JHEP 0610, 028 (2006). hep-ph/0608194

93. J.M. Campbell, R.K. Ellis, C. Williams, Phys. Rev. D 81, 074023 (2010). arXiv: 1001.4495 [hep-ph]

94. S. Catani, M. Grazzini, Phys. Rev. Lett. 98, 222002 (2007). hep-ph/0703012

95. M. Grazzini, JHEP 0802, 043 (2008). arXiv:0801.3232 [hep-ph]

96. M. Grazzini, H. Sargsyan, JHEP 1309, 129 (2013). arXiv:1306.4581 [hep-ph]

97. H.-L. Lai, M. Guzzi, J. Huston, Z. Li, P.M. Nadolsky, J. Pumplin, C.-P. Yuan, Phys. Rev. D 82, 074024 (2010). arXiv:1007.2241 [hep-ph]

98. A. Elagin, P. Murat, A. Pranko, A. Safonov, Nucl. Instrum. Meth. A 654, 481 (2011). arXiv:1012.4686 [hep-ex]

99. S. Gennai et al., [CMS collaboration], Eur. Phys. J. C 46(S01), 1 (2006)

100. F. Tarrade [ATLAS Collaboration], Nucl. Phys. Proc. Suppl. 169 357 (2007)

101. HL-LHC: High Luminosity Large Hadron Collider, http:// hilumilhc.web.cern.ch/HiLumiLHC/index.html 